\title{
Canonical Stochastic Realization of Turbulent Sound Sources via Forced Linear Advection-Diffusion-Dissipation Equation
}

\author{
R. Ewert* \\ Institute of Aerodynamics and Flow Technology, Technical Acoustics \\ Lilienthalplatz 7, 381208 Braunschweig, Germany
}

\begin{abstract}
Stochastic sound sources derived from Reynolds Averaged Navier-Stokes (RANS) solution are recognized in Computational Aeroacoustics as one possible way to efficiently predict broadband sound. In this paper a stochastically forced linear advection-diffusiondissipation equation is introduced. The model provides spectra and anisotropic two-point correlations that otherwise have to be incorporated in datum stochastic methods as additional model assumptions. The output are fluctuating velocity components, from which vortex sound sources derive. The forcing is white (delta-correlated) in time and possess a finite correlation length scale in space. The well-posedness is demonstrated in the paper. A solenoidal forcing term is shown to realize the correlation tensor of homogeneous isotropic turbulence together with a longitudinal turbulence spectrum that exhibits a plateau for lower frequencies followed by a characteristic power law roll-off and final cut-off. Exponent of decay and cut-off are adjustable. The stochastic partial differential equation involves a diffusion parameter, a time-scale, a re-distribution tensor, and a forcing variance. Transport equations for Reynolds stresses and turbulence kinetic energy derive from it that have the canonical form of major RANS transport equations. In particular, all parameters needed can be assigned to corresponding RANS parameters so that an accurate reproduction of RANS one-point statistics becomes feasible. For the generation of two-point statistics the hypothesis from turbulence modeling is adopted that the present model calibrated for homogeneous isotropic turbulence is also applicable for more general flows.
\end{abstract}

\section{Introduction}

In Computational Aeroacoustics (CAA) stochastic sound sources derived from Reynolds Averaged NavierStokes (RANS) solution are recognized as one possible way to establish fast and efficient prediction of broadband sound. Specific for all stochastic approaches used for CAA is that they have to provide continuous dynamics in space and time (3+1-D realizations) to prevent spurious noise sources.

Two main approaches for the stochastic generation of turbulent velocity fluctuations have been studied over the past years. Starting with the work of Kraichnan, ${ }^{1}$ one the one hand side random Fourier modes are used to prescribe wave-number spectra of turbulence. As a random Fourier mode method especially suited for CAA applications the stochastic noise generation and radiation (SNGR) method was introduced and developed further by Béchara et al. ${ }^{2}$ and Bailly \& Lafon. ${ }^{3,4}$

The second class of approaches used in CAA was introduced with the random particle-mesh (RPM) and fast random particle-mesh (FRPM) methods. ${ }^{5-8}$ These approaches utilize a numerical approximation of spatially distributed white-noise, which is filtered with a Gaussian filter of defined width and amplitude to generate fluctuations with locally prescribed cross-correlation function and variance.

Since the spatial cross-correlation function and the turbulence wave-number spectrum represent a Fouriertransform pair, a model realizing a specific turbulent cross-correlation function (two-point statistics) also provides the corresponding turbulence spectrum and vice versa.

*Senior Scientist, Senior Member AIAA, roland.ewert@dlr.de. 
In general, the goal of stochastic models is to obtain fluctuations that accurately reproduce key one-point statistics and parameters as provided by RANS, i.e. velocity fluctuations with levels in compliance with turbulence kinetic energy. Further information from RANS incorporated into the modeling of fluctuations are the length- and time scale as well as the RANS mean velocity to prescribe turbulence advection.

To incorporate a locally varying advection velocity field into a random Fourier mode representation of a wave-number spectrum is not straight forward. The direct replacement of wave-number $\boldsymbol{k} \boldsymbol{x}$ with $\boldsymbol{k}\left(\boldsymbol{x}-\boldsymbol{v}_{0} t\right)$ in all Fourier modes is only valid if the advection velocity $\boldsymbol{v}_{0}$ is spatially constant, i.e. uniform. An attempt to set-up a stochastic model based on a localized spectral description using wavelets has been discussed by Kozubskaya et al. ${ }^{9}$ For the consideration of non-uniform source advection, Billson et al. ${ }^{10}$ have proposed to combine a random Fourier mode description with an advection equation.

For stationary turbulent flow problems characterized by a highly non-uniform distribution of different target quantities (length- and time-scale, advection velocity, turbulence intensities), datum stochastic methods not always can achieve an unambiguous accurate simultaneous realization of all quantities.

This may allude to a general over-determination of the problem if all target features are aimed to be stochastically modeled as independent qualities, i.e., ignoring their mutual interconnection as established by the turbulence transport equations underlying the turbulence target statistics.

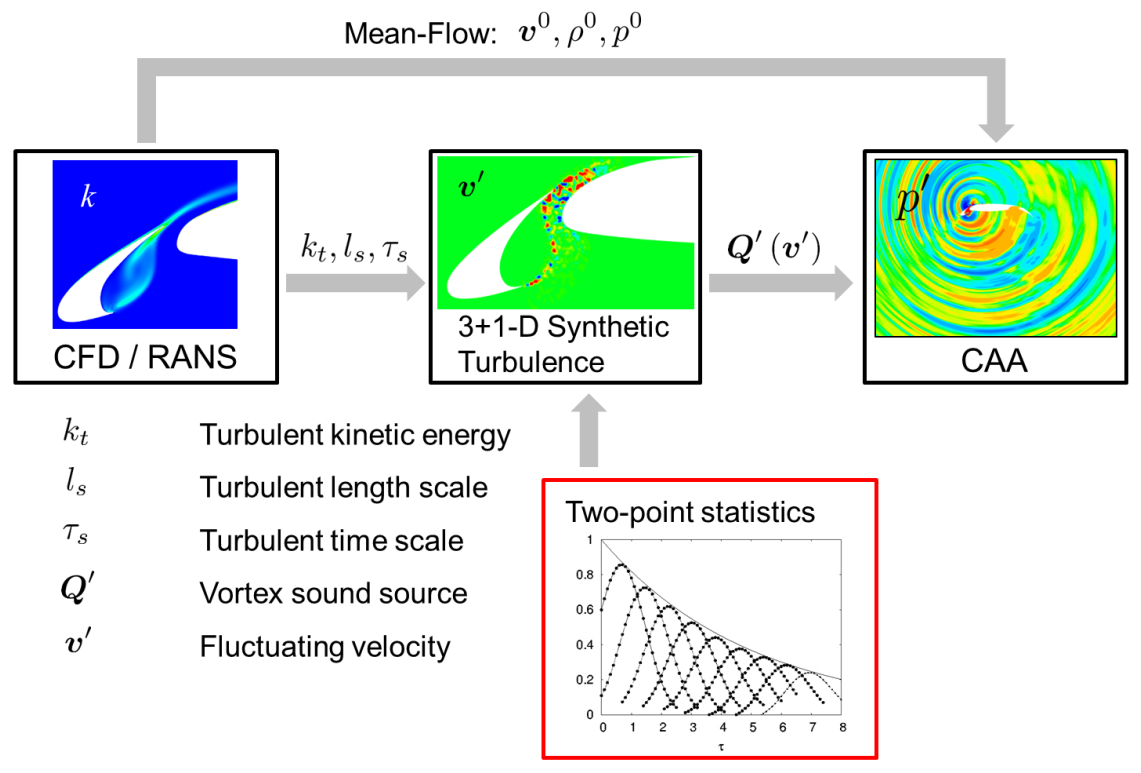

Figure 1. CFD/FRPM/CAA simulation procedure augmented by supplementary two-point information.

Furthermore, one-point RANS statistics in general provide an incomplete basis for source modeling as additional assumptions are needed to deduce sources with specific two-points statistics, e.g. assuming general validity of a specific turbulence spectrum or the specific shape of the cross-correlation function. Fig. 1 indicates the general problem exemplarily sketching the application of the FRPM method to high-lift slat noise: the one-point statistics have to be augmented by assumptions about the shape of the cross-correlation function to obtain sound sources with imposed two-point statistics.

The scalar length-scale parameter from RANS lacks the information about the anisotropy of the lengthscales. Therefore, in stochastic methods fluctuating velocity is mostly modeled assuming isotropic turbulence. Anisotropy in one-point statistics can be incorporated using RANS Reynolds stress models. However, although on an empirical basis attempts have been made to incorporate length-scale anisotropy into the modeling of velocity fluctuations, ${ }^{11,12}$ a empirical-free closed prediction of this extra information from RANS is missing. Alternatively, it has been proposed to take this additional piece of information from supplementary scale resolving large-eddy simulation. ${ }^{13}$

The consideration of anisotropic length scales is known to be significant for aeroacoustic predictions, refer to Kamruzzaman ${ }^{11}$ for the importance in the simulation of airfoil trailing edge noise from surface pressure statistics. For example, to predict surface pressure spectra from a simplified Poisson equation ${ }^{14,15}$ a more detailed description of the two-point statistics of the $v$-component normal to the wall are needed. ${ }^{11,12}$ 


\section{Outline}

In this work a linear advection diffusion dissipation equation forced by a stochastic source term will be introduced to accomplish velocity fluctuations with statistics in close agreement with target statistics provided by RANS. The approach enables the exploitation of more RANS parameters than usually used in stochastic models.

It is shown that the simulation approach provides spectra and two-point statistics. An initial calibration of the variance and the spectrum used in the stochastic forcing term provides the proper realization of the energy spectra of homogeneous isotropic turbulence (HIT). Furthermore, for HIT the methodology realizes solenoidal velocity fluctuations and provides an exact reproduction of the two-point velocity cross-correlation tensor.

Based on the hypothesis that the forcing spectrum is applicable also for more general cases, the linear advection-diffusion-dissipation equation in general provides synthetic turbulence entirely prescribed by the RANS statistics of inhomogeneous flow. Anisotropy in length scale is realized due to the deviation from equilibrium of the equation solved, refer to the qualitative discussion in Section III.

As a starting point, we aim to derive a model equation for turbulent velocity fluctuations whose variance equations agree with that of the corresponding RANS transport equations, i.e. with the Reynolds stresses or the resulting turbulent kinetic energy equation. For this purpose, the following forced linear advectiondiffusion-dissipation model equation will be closer studied in this work:

$$
\frac{\partial v_{i}^{\prime}}{\partial t}+v_{j}^{0} \frac{\partial v_{i}^{\prime}}{\partial x_{j}}-\frac{\partial}{\partial x_{j}}\left(D \frac{\partial v_{i}^{\prime}}{\partial x_{j}}\right)+\lambda_{i j} v_{j}^{\prime}=Q_{i}
$$

where superscript " 0 " indicates ensemble averaged quantities, i.e. $v_{i}^{0}=\langle v\rangle_{i}$, a prime denotes fluctuating velocity and $D$ is a diffusion parameter. The tensor $\lambda_{i j}$ has two contributions,

$$
\lambda_{i j}=\lambda \delta_{i j}+f_{i j}
$$

i.e. a diagonal part proportional to an inverse characteristic time scale $\lambda$ and a tensor $f_{i j}$ that will be defined later. A spatio-temporal stochastic forcing term $Q_{i}$ is introduced on the right-hand side of Eq. (1) to effectively model the production term $\hat{\mathcal{P}}$ in the resulting transport equation for turbulent kinetic energy. Straight forward evaluation of the turbulence kinetic energy $\left(k_{t}\right)$ equation related to Eq. (1) yields

$$
\frac{\partial k_{t}}{\partial t}+v_{j}^{0} \frac{\partial k_{t}}{\partial x_{j}}-\frac{\partial}{\partial x_{j}}\left(D \frac{\partial k_{t}}{\partial x_{j}}\right)=\hat{\mathcal{P}}-D\left\langle\frac{\partial v_{i}^{\prime}}{\partial x_{j}} \frac{\partial v_{i}^{\prime}}{\partial x_{j}}\right\rangle-2 \lambda k_{t}
$$

where the brackets indicate an ensemble average. The tensor $f_{i j}$ does not contribute to the turbulence kinetic energy budget but is important as it realizes the re-distribution tensor in the Reynolds stresses transport equation, Section V.B. Furthermore, the magnitude of the production term $\hat{\mathcal{P}}$ in this equation is defined by the correlation of the forcing term with the generated fluctuating velocity, viz

$$
\hat{\mathcal{P}}=\left\langle v_{i}^{\prime} Q_{i}\right\rangle,
$$

refer to the more detailed discussion provided by Section V.F.

The representative turbulence kinetic transport equation that is studied in the present paper is given by

$$
\frac{\partial k_{t}}{\partial t}+v_{i}^{0} \frac{\partial k_{t}}{\partial x_{j}}-\frac{\partial}{\partial x_{j}}\left(\left(\nu+\frac{\nu_{t}}{\sigma_{k}}\right) \frac{\partial k_{t}}{\partial x_{j}}\right)=\mathcal{P}-\epsilon,
$$

refer to Wilcox. ${ }^{16}$ Here $\epsilon$ denotes the pseudo dissipation,

$$
\epsilon=\nu\left\langle\frac{\partial v_{i}^{\prime}}{\partial x_{j}} \frac{\partial v_{i}^{\prime}}{\partial x_{j}}\right\rangle
$$

$\nu$ is the the kinematic viscosity, $\nu_{t}$ is the turbulent eddy viscosity, $\sigma_{k}$ indicates a modeling constant, and $\mathcal{P}$ denotes turbulence production. All quantities are determined if the turbulence kinetic energy transport equation is solved by a precursor RANS simulation. It is immediately clear that Eq. (3) corresponds with Eq. (5) if $D=\nu+\nu_{t} / \sigma_{k}$ and the right-hand side of Eq. (3) is adjusted via proper calibration of the forcing term $\hat{\mathcal{P}}$ and inverse time-scale $\lambda$ so that it complies with the right-hand side of Eq. (5). 
Consequently, if properly calibrated, Eq. (1) formally enables a reproduction of turbulence kinetic energy (one-point statistics) from RANS. However, in order to demonstrate the capability of Eq. (1) to reproduce in addition also meaningful turbulence spectra and two-point statistics the following questions must be answered:

- Is the solution of a diffusion-convection equation with stochastic source term at all well posed?

- How has the variance of the stochastic production term to be calibrated to accomplish a specific locally prescribed turbulent kinetic energy production?

- How should the spectrum of the forcing term be defined to obtain a specific equilibrium spectrum?

- Is the energy cascade of 3-D turbulence from large to small scales correctly recovered by the model?

The previous questions are furthermore accompanied by questions of application, e.g. the proper numerical realization of the previous sketched model equation. In particular, for applications typically fluctuations are only resolved up to a coarse cut-off wave-number, far above the Kolmogorov length scale. Hence, the calibration of constants might be further affected by the truncation of scales.

A brief qualitative discussion on the realizability of anisotropic length scales is provided by the next paragraph. The derivation of a forced linear advection-diffusion-dissipation equation as a proper way to realize realistic turbulence spectra and homogeneous isotropic turbulence is discussed in Section IV using some FRPM key features. Section V deals with generalization of the model to comply with standard RANS transport equations. Section VII gives a brief overview about some numerical results obtained with the approach. A summary with conclusions is given in Section VIII.

\section{Phenomenological discussion}

For homogeneous isotropic turbulence the integral length scale provided by Eq. (1) results from a dynamic process in equilibrium, where diffusion and dissipation are balanced by corresponding production.

Main characteristic variables of this process are the diffusion parameter $D$ and the time scale $1 / \lambda$ in Eq. (1). On dimensional grounds, the length scale results from $l_{s}=\sqrt{D / \lambda}$. Closer scrutiny reveals that the inverse time scale must be calibrated to $\lambda \propto 1 / \tau_{s}$ with the turbulent time-scale defined from RANS quantities,

$$
\tau_{s}=c_{\tau} \frac{k_{t}}{\epsilon} .
$$

As discussed in the previous section, the RANS eddy viscosity

$$
\nu_{t}=C_{\mu} \frac{k_{t}^{2}}{\epsilon}
$$

(with constant $C_{\mu}=0.09$ ) is the main contributor to the diffusion constant, i.e., $D=\nu_{t} / \sigma_{k}$. Consequently, a characteristic length-scale from combination of diffusion parameter and time-scale infers as

$$
l_{s}=c_{l} \frac{k_{t}^{3 / 2}}{\epsilon},
$$

where $c_{l}$ is a order one parameter that effectively results from the modeling parameters used. The length scale is in correspondence with the definition generally used in stochastic modeling.

Anisotropy in length scale is present in most technical relevant flows. The length-scale scalar from RANS usually is interpreted to result from a suitable contraction of the actual anisotropic length scale tensor, e.g. as being discussed by Rotta, ${ }^{17}$ refer to Eq. (4.55) in Wilcox. ${ }^{16}$

For a phenomenological discussion of the anisotropic length scales resulting from Eq. (1) we consider the problem of turbulence seeded upstream of a NACA 0012 airfoil as discussed by Wohlbrandt et. al. ${ }^{18}$ From RANS simulation and Eq. (9) a typical length scale distribution as shown in Fig. 2 results. ${ }^{18}$ In this example a SST $k_{t}-\omega$ turbulence closure is applied (the dissipation rate is related to the specific dissipation rate $\omega$ from the model by $\left.\epsilon=C_{\mu} k_{t} \omega\right)$.

It can be observed that along the symmetry line upstream of the airfoil leading edge the turbulence length scale of the seeded turbulence is constant, but drops down rapidly to zero when approaching the 


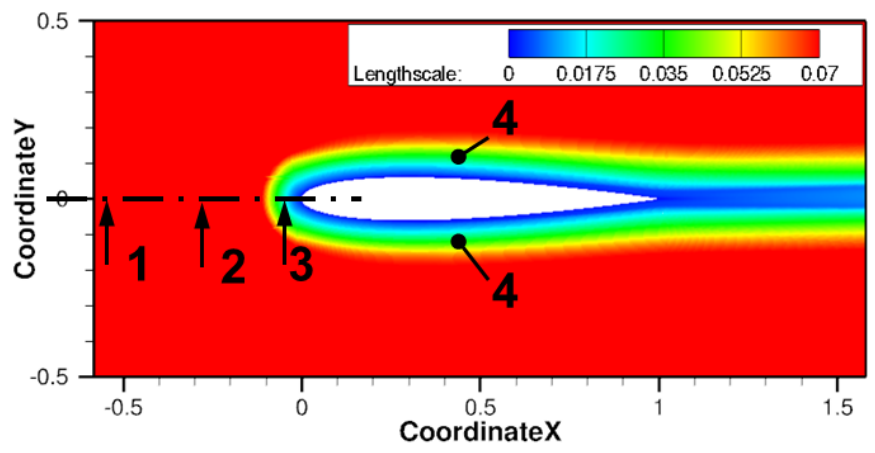

(a) A.

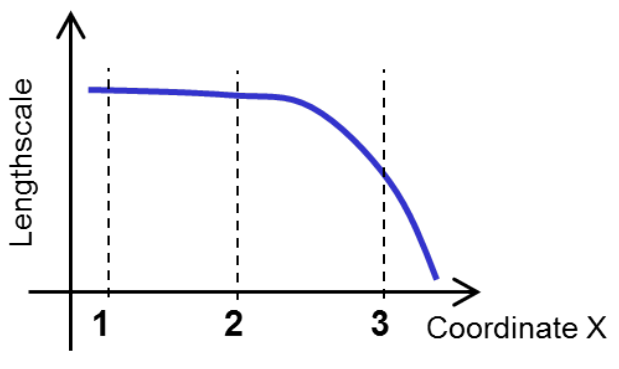

(b) B.

Figure 2. RANS length scale distribution Eq. (9) resulting from turbulence seeded upstream of an airfoil. ${ }^{18}$

airfoil leading edge. This behavior is furthermore highlighted in the sketch Fig. $3^{18}$ showing the dynamics of a vortex of characteristic eddy size representing the local present length scales in a sequence. In the lower sketch the length-scale development from RANS is indicated, which would result from direct application of Eq. (9). The upstream vortex size decreases to zero when reaching the airfoil stagnation point. However, expected would be a vortex dynamic as indicated by the upper sketch. Note, the decreasing length-scale in the lower sketch would be expected on grounds of the effective averaging (contracting) procedure used to determine the scalar length-scale parameter from the anisotropic scales. For example, in position 3 before the airfoil the average of the streamwise and crosswise length-scales yields a reduced effective scalar length scale in qualitative agreement with the lower sketch.
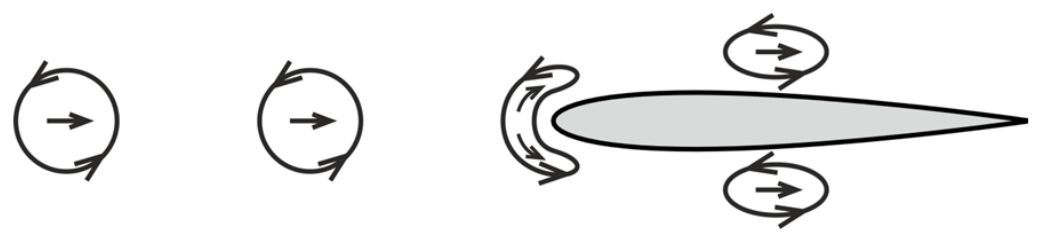

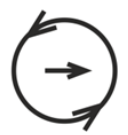

1

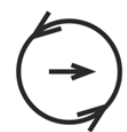

2

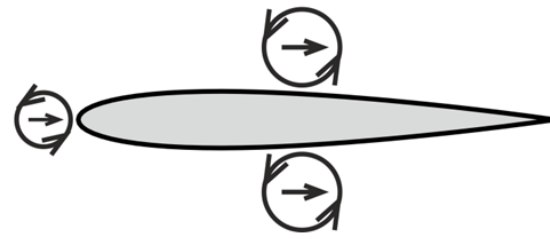

3

4

Figure 3. Sketch of the development of characteristic length-scales for the upstream turbulence problem; expected anisotropic behavior, (top); RANS scalar length-scale applied to model isotropic turbulence, (bottom).

Concerning a stochastic realization of the upstream turbulence problem with Eq. (1), from a Lagrangian frame perspective close to the leading edge of the airfoil the dynamic process would be away from equilibrium, characterized by a rapid change of time scale and diffusion parameter. The resulting length scale develops as a consequence of its previous history and the spatial changes in diffusion, dissipation, and production. Close to the leading edge the length scales would be anisotropic. Specifically, in position 3 before the airfoil leading edge the reduction of the diffusion constant and time scale in flow direction compared to the corresponding values along a line perpendicular to the flow qualitatively results in a reduced length scale in flow direction, while the scale perpendicular to the flow may remain mainly unchanged, i.e. in correspondence with the characteristic shown in the upper sketch of Fig. 3.

Note, the previous example indicates the possible to realize additional features of turbulence, e.g., details of the length-scale anisotropy if the dynamics as encoded in the RANS turbulence transport equations are 
also incorporated into the stochastic realization. This is explicable on the grounds that if target quantities obey a governing transport equation, a mutual dependencies is established among them that can determine additional characteristics of otherwise not explicit modeled yet dependent qualities.

\section{General turbulence spectra via F.L.A.D.D.}

In the following paragraph the key features of $\mathrm{FRPM}^{5-8,19}$ are introduced ${ }^{\mathrm{a}}$ and utilized to discuss the realizability of turbulence spectra from a forced linear advection-diffusion-dissipation (F.L.A.D.D.) equation.

\section{A. Basic FRPM tool kit}

The Fast Random Particle-Mesh (FRPM) method has been introduced in previous work as a method to generate stochastic sound sources for CAA. ${ }^{5-8,19}$ It has been successfully applied to various broadband noise problems. ${ }^{20-22}$ However, the problems as discussed in the Introduction also hold for FRPM and motivate a further development towards canonical stochastic realization. Eventually, FRPM will be used to set up the resulting F.L.A.D.D. forcing term.

With the FRPM approach a fluctuating vector field $q_{i}$ is generated from the spatial convolution of stochastic fields $\mathcal{U}_{i}$ applying a Gaussian filter,

$$
q_{i}(\boldsymbol{x}, t)=\int G\left(\left|\boldsymbol{x}-\boldsymbol{x}^{\prime}\right|, l\right) \mathcal{U}_{i}\left(\boldsymbol{x}^{\prime}, t\right) \mathrm{d}^{3} \boldsymbol{x}^{\prime},
$$

or, in brief notation,

$$
q_{i}=G \star \mathcal{U}_{i}
$$

The Gaussian filter is given by

$$
G(r, l)=\exp \left(-\frac{\pi r^{2}}{2 l^{2}}\right),
$$

where $l$ represents the realized length-scale. The cross-correlation function that is generated by this procedure is proportional to the self-convolution of the Gaussian. This yields also a Gaussian cross-correlation function and Gaussian energy spectra ${ }^{\mathrm{b}}$, refer to the more detailed discussion in Appendix A.1.

To model turbulent advection together with an exponential turbulent decay at time scale $\tau_{s}$, the stochastic fields $\mathcal{U}_{i}$ are defined in a local frame of reference moving at velocity $v_{i}^{0}$ by means of a Langevin equation (Lagrangian coordinates indicated by double prime)

$$
\frac{\partial}{\partial t} \mathcal{U}_{i}\left(\boldsymbol{x}^{\prime \prime}, t\right)+\frac{1}{\tau_{s}} \mathcal{U}_{i}\left(\boldsymbol{x}^{\prime \prime}, t\right)=\sqrt{\frac{2}{\tau_{s}}} \xi_{i}\left(\boldsymbol{x}^{\prime \prime}, t\right)
$$

The advection velocity usually is taken from the mean-flow field from RANS. The components of the source term $\xi_{i}\left(\boldsymbol{x}^{\prime \prime}, t\right)$ are white in space and time, mutually uncorrelated, and have vanishing mean. Their definition in the Lagrangian frame reads

$$
\begin{aligned}
\left\langle\xi_{i}\left(\boldsymbol{x}^{\prime \prime}, t\right)\right\rangle & =0 \\
\left\langle\xi_{i}\left(\boldsymbol{x}^{\prime \prime}, t\right) \xi_{j}\left(\boldsymbol{x}^{\prime \prime}+\boldsymbol{r}, t+\tau\right)\right\rangle & =c\left(\boldsymbol{x}^{\prime \prime}\right) \delta(\boldsymbol{r}) \delta(\tau) \delta_{i j} .
\end{aligned}
$$

The field $c$ defines the proper variance of the target quantities $q_{i}$. Its scaling is given in Appendix A.2.

The properties of the source imply also a spatial definition of $\mathcal{U}_{i}$, explicitly indicated in Eq. (13) by the partial time derivative and dependence on $\boldsymbol{x}^{\prime \prime}$. Otherwise, since the left-hand side of Eq. (13) is spatially decoupled, it completely complies with an ordinary Langevin equation.

For the quadrature of the integral in Eq. (10) the computational domain is formally split into equal sized non-overlapping control volumes $\delta V_{k}$ of constant size, continuously covering the resolved source domain without holes. A random particle is assigned to the center of each control volume $\boldsymbol{x}_{k}$. All resulting particles

\footnotetext{
additional information is provided by Appendix A.

b The shape of cross-correlation function to be Gaussian is specified in FRPM approach as an extra modeling assumption. An extension to general turbulence spectra (and their corresponding cross-correlation functions) is given in Appendix A.3. However, this specific choice and general application is also a modeling assumption. Furthermore, it relies on a definition of one length-scale scalar as given by Eq. (9), i.e. an anisotropy in length scales is not recognized.
} 
are moving with their local advection velocity at the particle location. The control volumes are bounded by liquid-line surfaces, i.e. the boundary surface is drifting with the flow and in incompressible flow the control volume $\delta V_{k}$ is invariant over time. The extension of the procedure to compressible flow was discussed in Ewert et al., 8 but is not considered here. Random variables are attached to each particle. Their random variates $^{\mathrm{c}}$ at time level $t^{n+1}$ formally are defined by the integral over the control volume of the stochastic fields at the current time level, i.e.,

$$
r_{i k}^{n+1}:=\int_{\delta V_{k}} \mathcal{U}_{i}\left(\boldsymbol{x}^{\prime \prime}, t^{n+1}\right) \mathrm{d}^{3} \boldsymbol{x}^{\prime \prime} .
$$

Eventually, the convolution Eq. (10) is approximated at discrete time level $n+1$ by summation over all control volumes,

$$
q_{i}^{n+1}(\boldsymbol{x}) \simeq \sum_{k} G\left(\left|\boldsymbol{x}-\boldsymbol{x}_{k}\right|, l\right) r_{i k}^{n+1} .
$$

Subsequently, the discrete convolution operation will be indicated by a modified asterisk-symbol,

$$
q_{i}^{n+1}=G \widehat{\star} r_{i k}^{n+1}
$$

Numerically, only random variables $r_{i k}$ are realized and the stochastic field $\mathcal{U}_{i}$ serves only to facilitate the derivation of a discretized random process to generate the actual variates of each random variable.

From Eq. (16) together with Eq. (13) one Langevin equation for each random variate derives, ${ }^{8}$ whose discretized form reads $^{23}$

$$
r_{i k}^{n+1}=\left(1-\frac{\Delta t}{\tau_{s}}\right) r_{i k}^{n}+\left(\frac{2 c \delta V_{k} \Delta t}{\tau_{s}}\right)^{1 / 2} \sigma_{i k}^{n},
$$

where $\sigma_{i k}^{n}$ are mutually uncorrelated standardized Gaussian random variates, which are independent of themselves at different times $\left(\left\langle\sigma_{i k}^{n}\right\rangle=0,\left\langle\sigma_{i k}^{n} \sigma_{j l}^{m}\right\rangle=\delta_{i j} \delta_{k l} \delta_{n m}\right)$, and which are independent of $r_{i k}^{m}$ at past times (e.g., $\left\langle\sigma_{i k}^{n} r_{j l}^{m}\right\rangle=0$ for $t^{m} \leq t^{n}$. The time increment between time levels $t^{n+1}$ and $t^{n}$ is given by $\Delta t<\tau_{s}$.

Hence, one simulation step to proceed form time level $t^{n}$ to $t^{n+1}$ consists of the following operations:

- move the particles from old position at time level $n$ to the new one at time level $n+1$,

- realize with Eq. (19) new variates of all random variables attached to each particle,

- distribute random variates via discretized convolution Eq. (18) onto the source domain.

A discussion of the methods applied for the seeding of particles at inflow boundaries (and removal at outflow boundaries) and the efficient realization of the discrete convolution via recursive Gaussian filters can be found in Refs. 8,19

Note, by recursion, the discretized Langevin equation (19) can be rewritten as the summation of all source variates over all previous time levels, i.e., using the definitions

$$
\alpha:=\left(\frac{2 c \delta V_{k} \Delta t}{\tau_{s}}\right)^{1 / 2}, \quad \beta:=1-\frac{\Delta t}{\tau_{s}},
$$

it follows

$$
r_{i k}^{n+1}=\alpha \sigma_{i k}^{n}+\alpha \beta \sigma_{i k}^{n-1}+\alpha \beta^{2} \sigma_{i k}^{n-2}+\alpha \beta^{3} \sigma_{i k}^{n-3}+\alpha \beta^{4} \sigma_{i k}^{n-4}+\cdots \cdots+\beta^{n+1} r_{i k}^{0} .
$$

Since $\beta<1$, for $n+1$ sufficiently large, the contribution of the last term will become negligible.

\footnotetext{
'The notion 'random variate' is used here to indicate the specific value of a random variable at a specific time level, e.g. random variates $r_{i k}^{n+1}$ indicate the value of random variables $r_{i k}$ at time level $n+1$.
} 


\section{B. From FRPM to F.L.A.D.D.}

\section{Forced linear advection-dissipation equation}

The principal FRPM procedure to compute a stochastic field for a new discrete time level as outlined in the previous section is symbolically indicated in Fig. 4(a) for one random variable and particle. To simplify notation, the double index ' $i k$ ' for component and particle identification has been dropped in this and the subsequent figures. As shown by Eq. (21), the random variates of the discretized Langevin equation consist of the weighted sum of all stochastic source terms at previous time levels. That is, effectively first all source variates $\sigma^{n-m}$ are weighted with $\alpha \beta^{m}$ and summed up. Next, the resulting variate $r^{n+1}$ is distributed with a Gaussian into the neighborhood of the present particle to generate the new fluctuating quantity $q^{n+1}$.

In the full approach distributed particles are used to generate a fluctuating vector potential with components $q_{i}^{n+1}$. A velocity field is obtained by taking the curl of $q_{i}$, refer to Eq. (103) in Appendix A.2. The spatial cross-correlation function $\mathcal{R}_{i j}=\left\langle v_{i}^{\prime} v_{j}^{\prime}\right\rangle$ that finally results from the full procedure is based on a Gaussian longitudinal correlation function (Appendix A.1); the resulting correlation function is also sketched in Fig. 4(a).

In principle, it is possible to obtain the same filtered field if the order of discrete convolution and weighted summation over random variates is exchanged, i.e., implying an exchange of convolution and Langevin procedure. This is indicated in Fig. 4(b). The summation goes over the Gaussian fields obtained from the convolution of the random variates at each time level. The width of the Gaussian at all time levels is fixed, however, the amplitude successively decays due to the scaling with factors $\alpha \beta^{m}$. Note, the sketch only shows shapes of the weighted Gaussian filter $\alpha \beta^{m} G$.

This exchange also can be applied to the non-discretized Langevin equation, Eq. (13) with convolution Eq. (11). Applying the convolution to Eq. (13), exchanging the order of differentiation and convolution (filter with constant length scale applied), and transforming back into a fixed coordinate system, a forced advection-dissipation equation for the target quantity is obtained, i.e.,

$$
\frac{\partial q_{i}}{\partial t}+v_{j}^{0} \frac{\partial q_{i}}{\partial x_{j}}+\frac{1}{\tau_{s}} q_{i}=W_{i}
$$

Note, from its definition, Eq. (10), it follows that the field $q_{i}$ is continuous differentiable in space. The resulting right-hand side white-noise term infers from

$$
W_{i}=\sqrt{\frac{2 c\left(\boldsymbol{x}^{\prime \prime}\right)}{\tau_{s}}}\left(G \star \xi_{i}\right) .
$$

From Eqs. (14) and (15), which define $\xi_{i}$ in a Lagrangian frame moving at advection velocity, the Lagrangian properties of the forcing term can be deduced (for the derivation of the properties refer to the discussion, e.g., in $\left.\operatorname{Ref}^{8}\right)$,

$$
\begin{aligned}
\left\langle W_{i}\left(\boldsymbol{x}^{\prime \prime}, t\right)\right\rangle & =0, \\
\left\langle W_{i}\left(\boldsymbol{x}^{\prime \prime}, t\right) W_{j}\left(\boldsymbol{x}^{\prime \prime}+\boldsymbol{r}, t+\tau\right)\right\rangle & =\frac{2 c\left(\boldsymbol{x}^{\prime \prime}\right)}{\tau_{s}\left(\boldsymbol{x}^{\prime \prime}\right)} R(\boldsymbol{r}) \delta(\tau) \delta_{i j},
\end{aligned}
$$

where $R=G \star G$ is given by Eq. (102). That is, the source term is white in time but spatially continuous with Gaussian cross-correlation function.

In the subsequent discussion we start first by assuming a constant mean-flow $v_{i}^{0}=$ const. A stochastic differential equation for fluctuating velocity can be obtained by taking the curl of Eq. (22), assuming $q_{i}$ to represent a vector potential from which the velocity derives via $v_{i}^{\prime}=\epsilon_{i j k} \partial q_{k} / \partial x_{j}$, refer to Eq. (103) in the Appendix. This yields (based on a constant mean-flow velocity)

$$
\frac{\partial v_{i}^{\prime}}{\partial t}+v_{j}^{0} \frac{\partial v_{i}^{\prime}}{\partial x_{j}}+\frac{1}{\tau_{s}} v_{i}^{\prime}=\epsilon_{i j k} \frac{\partial W_{k}}{\partial x_{j}},
$$

i.e. the velocity fluctuations as defined by Eq. (103) are generated by means of a forced advection-dissipation equation. Otherwise, all properties of the genuine FRPM method as discussed in Appendix A.2 are preserved. In particular, for homogeneous isotropic turbulence (HIT) the solution $v_{i}^{\prime}$ realizes the correlation tensor of HIT,

$$
\mathcal{R}_{i j}(\boldsymbol{r}, \tau)=v_{t}^{2}\left[\frac{f(r)-g(r)}{r^{2}} r_{i} r_{j}+g(r) \hat{\delta}_{i j}\right]
$$


(a)

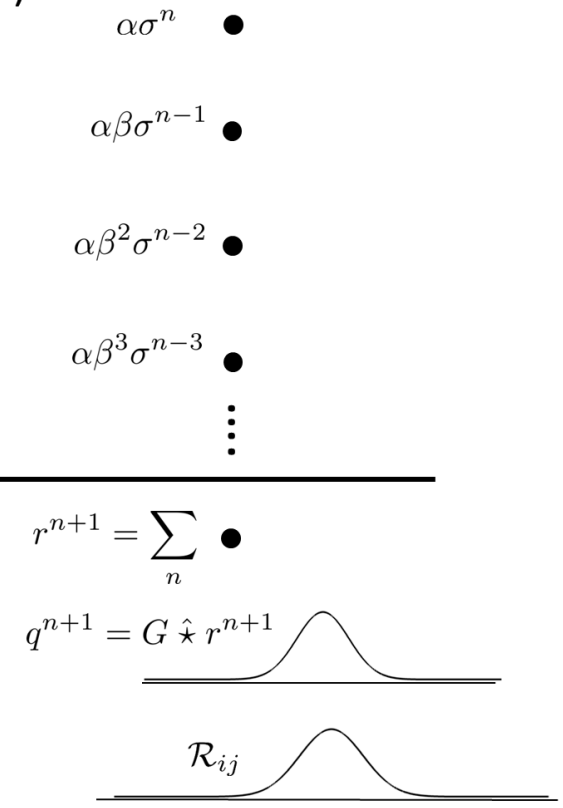

(b)
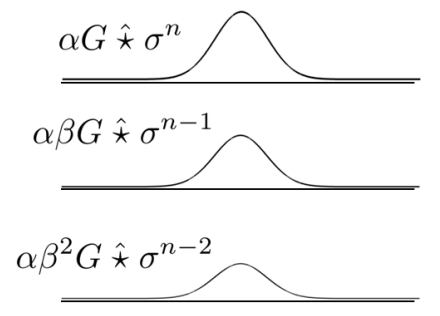

$\alpha \beta^{3} G \hat{\star} \sigma^{n-3}$

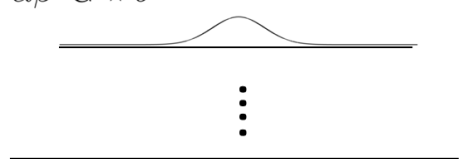

$$
q^{n+1}=\sum_{n}
$$

$q^{n+1}$

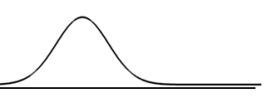

$\mathcal{R}_{i j}$

Figure 4. Generation of decaying turbulence with FRPM (Gaussian spectrum); (a), superposition of random variates at different time levels as effectively realized by discretized Langevin equation (19) and subsequent discrete convolution with Gaussian filter, Eq. (18); (b), exchanging order of superposition and discrete convolution yields same result (only effective filter shapes $\alpha \beta^{m} G$ sketched).

(a)

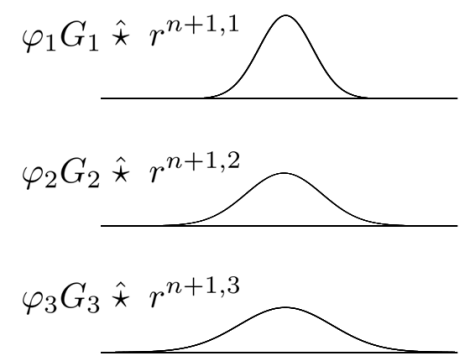

$$
\varphi_{4} G_{4} \hat{\star} r^{n+1,4}
$$
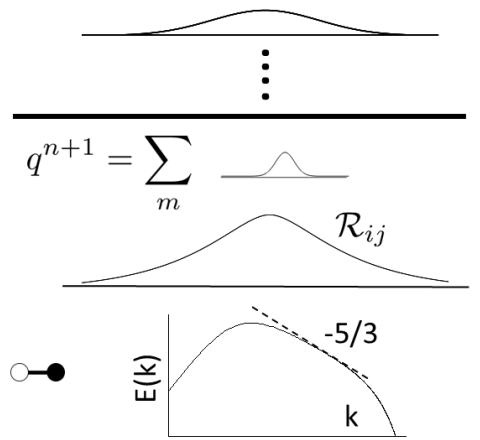

(b)
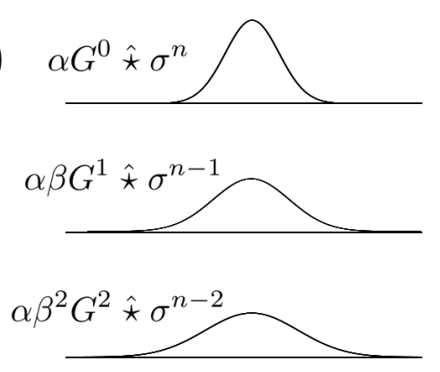

$\alpha \beta^{3} G^{3} \hat{\star} \sigma^{n-3}$
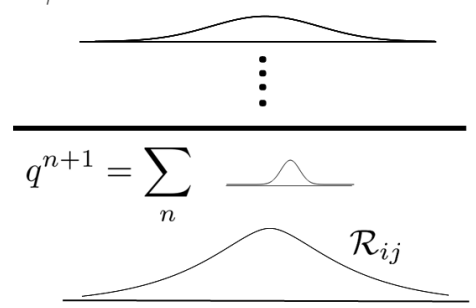

Figure 5. Generation of arbitrary turbulence spectra; (a), realization via superposition of filtered and weighted mutually uncorrelated random variates $r^{n+1, m}$ using Gaussian filter of successively increased filter width (discrete Gaussian transformation ${ }^{24,25}$ ); (b), same summation of time-levels as in Fig. $4(\mathrm{~b})$ but with the initial Gaussian filter $G^{0}$ developing according to a diffusion equation; only effective filter shapes $\varphi_{m} G_{m}$ and $\alpha \beta^{m} G^{m}$ sketched. 
where $r=|\boldsymbol{r}|$ and $r_{i}$ indicates a component of $\boldsymbol{r}$ and $v_{t}^{2}$ is the turbulence intensity. Furthermore, $g(r)$ is the lateral and $f(r)$ is the longitudinal correlation function. In 2-D the resulting lateral correlation function is related to the longitudinal correlation function via

$$
g(r)=f(r)+r \frac{\mathrm{d} f(r)}{\mathrm{d} r},
$$

in 3 -D it becomes

$$
g(r)=f(r)+\frac{r}{2} \frac{\mathrm{d} f(r)}{\mathrm{d} r} .
$$

The source term occurs in Eq. (26) as a curl of a vector. We denote this type of forcing as solenoidal forcing (SF), whereas the forcing used in Eq. (22) is denoted as non-solenoidal forcing (NSF).

\section{Effect of diffusion added}

The turbulence spectra generated with FRPM are Gaussian, refer to the discussion in Appendix A.1. The generation of generalized turbulence spectra has been discussed in recent work by Rautmann et al. ${ }^{25}$ and Wohlbrandt et al. ${ }^{24}$ Details of the approach as far as they concern the present discussion are presented in Appendix A.3.

Effectively, standard turbulence spectra are generated through the weighted summation of $M$ independent FRPM realizations of Gaussian spectra with successively increased length scale. The numerical procedure conducted for every computational step is indicated in Fig. 5(a).

There, $G_{m}=G\left(r, l_{m}\right)$ denotes a Gaussian filter of length scale $l_{m}, \varphi_{m}$ indicates the weighting, and $r^{n+1, m}$ the random variate of the considered particle at time level $n+1$ (again, one particle considered and index $i k$ dropped for convenience). The second upper case index indicates the summation index of the random variate related to the generation of Gaussian spectrum $m \in\{1 \ldots M\}$. All random variates are generated by means of the discrete Langevin procedure Eq. (19) as mutually independent quantities, i.e.

$$
\left\langle r^{n+1, m} r^{n+1, l}\right\rangle=\delta_{m l} .
$$

The length scale is defined by $l_{m}=l_{0} q^{m}$, where $l_{0}$ is the smallest resolved scale. However, different monotonic functions to determine the length scale of realization $m$ could be chosen. The actual choice affects the definition of function $\varphi_{m}=\sqrt{h\left(l_{m}, \Lambda\right) \Delta l_{m}}$, where $\Delta l_{m}$ defines an increment between consecutive length scales and $h\left(l_{m}, \Lambda\right)$ is the generating function for a specific spectrum of integral length scale $\Lambda$. Analytical expressions of the generating function $h(l, \Lambda)$ has been given in recent work for a Liepmann spectrum, ${ }^{25}$ refer also to Eq. (112), as well as the von Kármán and modified von Kármán spectrum. ${ }^{24}$ Further details can be found in Appendix A.3.

As indicated in Fig. 5(a), the superposition of Gaussian filtered fields yields a cross-correlation function of velocity fluctuations $\mathcal{R}_{i j}$ that eventually provides generalized turbulence spectra via Fourier-transform. The last step is symbolically indicated in the figure with a Fourier-transform symbol.

Note, based on a set of actual variates at time level $n+1$, the procedure provides the desired wave-number spectra. In addition, turbulence decay still can be modeled as an independent quality using a time scale $\tau_{s}$ in the discrete Langevin equation.

The procedure shown in Fig. 5(b) is analog to Fig. 4(b), i.e. it shows first filtered then weighted timelevels of the source term Eq. (21). However, in this case the width of the Gaussian filter is not fixed but rather develops as prescribed by a diffusion equation, i.e. the Gaussian shape and the value of its spatial integral are conserved, causing a continuous decrease of the Gaussian peak amplitude, accompanied by a successively increased spatial width. The time-evolution of the filter is indicated by successively increasing upper case indices, starting with $G^{0}$ (recall that every level indicated in Fig. 5(b) represents a discrete past time level). Further note, similar to Eq. (30) the random variates used as sources in the discretized Langevin equation are also mutually uncorrelated, i.e. satisfy

$$
\left\langle\sigma^{n} \sigma^{m}\right\rangle=\delta_{n m} .
$$

Together with this feature, the procedure shown in Fig. 5(b) is formally equivalent to that applied in Fig. 5(a). This indicates the principle possibility to generate with added diffusion general spectra of turbulence, similar to Fig. 5(a). However, in case of Fig. 5(b) the actual generating function cannot be freely chosen but is fixed by the characteristics of the diffusion process and weighting coefficients from the discretized Langevin 
equation, $\alpha \beta^{m}$. However, it should be noted that the initial filter function $G^{0}$ could be calibrated itself as a weighted sum of Gaussians of different length scales. By generation of an appropriate forcing spectrum through the superposition of Gaussians ${ }^{24}$ it is feasible to accomplish the desired HIT target velocity spectrum.

In terms of the previous discussion of the transport equation (26), it must be augmented by an additional diffusion term,

$$
\frac{\partial v_{i}^{\prime}}{\partial t}+v_{j}^{0} \frac{\partial v_{i}^{\prime}}{\partial x_{j}}-D \frac{\partial^{2} v_{i}^{\prime}}{\partial x_{j}^{2}}+\frac{1}{\tau_{s}} v_{i}^{\prime}=\epsilon_{i j k} \frac{\partial W_{k}}{\partial x_{j}} .
$$

As indicated in the previous paragraph, the field induced by the forcing in Eq. (26) is continuous differentiable in space, so that adding an extra diffusion term does not pose any mathematical problem. Note, the previous discussion of Fig. 5(b) was based on a surgical decomposition of the numerical approach realized with FRPM, i.e. considering one particle (local forcing) and decomposing of the random variates therein explicitly into their past components. Eq. (32) is an equivalent formulation of this numerical approach, formulated as a stochastic partial differential equation (SDE). Since this SDE is linear, the superposition principle applies. That is, it 'automatically' supports the proper dynamics of all the past dynamics encoded in the solution as well as that of distributed forcing.

The forced linear advection-diffusion-dissipation (F.L.A.D.D.) equation (32) provides not only target wave-number spectra but also includes turbulence decay, i.e. both properties are not modeled independently but follow as a result of the modeling approach.

Furthermore, the modifications applied to Eq. (32) in comparison to Eq. (26), i.e. adding the diffusion term and using an effective forcing derived from a superposition of single forcing terms, still yields the cross-correlation tensor of homogeneous isotropic turbulence, i.e. the relationships Eq. (27) with Eq. (28) or Eq. (29) are conserved, refer to the more detailed discussion in Section V.G.2 below.

\section{Calibration with RANS transport models}

\section{A. RANS transport equations}

Subsequently the general form of the stochastic differential equation for general non-uniform flow and its proper scaling from RANS transport equations is elaborated. As a first step, only turbulence transport equations in the incompressible limit are considered. The corresponding Reynolds Averaged Navier-Stokes (RANS) equations for incompressible flow derive by decomposition of the flow variables into Reynolds (ensemble) averaged mean-flow and fluctuating parts,

$$
v_{i}=v_{i}^{0}+v_{i}^{\prime}, \quad p=p^{0}+p^{\prime}
$$

where superscript " 0 " indicates ensemble averaged quantities, i.e. $v_{i}^{0}=\langle v\rangle_{i}$, and a prime denotes the fluctuating part (incompressibility implies $\rho=\rho^{0}=$ const).

In the RANS equations governing the mean-flow part also the Reynolds stress tensor $\mathcal{R}_{i j}=-\left\langle v_{i}^{\prime} v_{j}^{\prime}\right\rangle$ occurs. In order to have a closed system it has to be appropriately modeled from resolved time-averaged flow quantities. For example, using the Boussinesq assumption, ${ }^{16}$ the Reynolds stresses are modeled from a turbulent eddy viscosity $\nu_{t}$, in combination with the mean-flow strain $S_{i j}=1 / 2\left(\partial v_{i}^{0} / \partial x_{j}+\partial v_{j}^{0} / \partial x_{i}\right)$ and the turbulence kinetic energy $k_{t}=\left\langle{v_{i}^{\prime}}^{2} / 2\right\rangle$,

$$
\mathcal{R}_{i j}=2 \nu_{t} S_{i j}+\frac{2}{3} \delta_{i j} k_{t} .
$$

The turbulent eddy viscosity has to be expressed through resolved mean-flow quantities. For example, in the standard $k_{t}-\epsilon$-model, ${ }^{16}$ a transport equation for the turbulent kinetic energy $k_{t}$ and its dissipation rate $\epsilon$ is solved, from which the turbulent eddy viscosity is deduced according to Eq. (8).

The turbulence kinetic energy equation is derived from the equation governing the fluctuations of velocity. Using variable decomposition Eq. (33), the equation that governs velocity fluctuations reads

$$
\underbrace{\frac{\partial v_{i}^{\prime}}{\partial t}+v_{j}^{0} \frac{\partial v_{i}^{\prime}}{\partial x_{j}}+v_{j}^{\prime} \frac{\partial v_{i}^{0}}{\partial x_{j}}+\frac{1}{\rho^{0}} \frac{\partial p^{\prime}}{\partial x_{i}}-\nu \frac{\partial^{2} v_{i}^{\prime}}{\partial x_{j}^{2}}+\frac{\partial}{\partial x_{j}}\left(v_{i}^{\prime} v_{j}^{\prime}-\left\langle v_{i}^{\prime} v_{j}^{\prime}\right\rangle\right)}_{\mathcal{N}_{i}}=0 .
$$


Transport equations for the Reynolds stresses are derived by evaluation of the identity

$$
\left\langle v_{i}^{\prime} \mathcal{N}_{j}+v_{j}^{\prime} \mathcal{N}_{i}\right\rangle=0 .
$$

The governing equation for the turbulence kinetic energy are deduced from this equation by contraction of indices and multiplication with $1 / 2$, respectively, using the identity

$$
\left\langle v_{i}^{\prime} \mathcal{N}_{i}\right\rangle=0 .
$$

The $k_{t}$-equation derived from the previous program leads to the well-known closure problem as it posses correlations not directly expressed in terms of resolved quantities. Therefore, the closure pursued in RANS is based on a model $k_{t}$-equation expressed in terms of advection, diffusion, production and dissipation terms, i.e. Eq. (5).

Accompanied with a transport equation for the pseudo-dissipation $\epsilon$, Eq. (6), (or specific dissipation rate $\omega)$, and using the Boussinesq assumption Eq. (34) together with the definition of the eddy viscosity Eq. (8), a closed formulation of the Reynolds stresses is accomplished.

Alternatively, transport equations for all 6 independent Reynolds stress components have been proposed, which formally can be written as

$$
\frac{\partial \mathcal{R}_{i j}}{\partial t}+v_{k}^{0} \frac{\partial \mathcal{R}_{i j}}{\partial x_{k}}=\mathcal{P}_{i j}-\epsilon_{i j}+\mathcal{D}_{i j}+\Pi_{i j} .
$$

On the right-hand side production, dissipation, and diffusion terms for each Reynolds stress component occur (buoyancy and rotation effects not considered). Furthermore, $\Pi_{i j}$ indicates the re-distribution tensor that enforces the re-establishment of isotropic turbulence, however does not change the turbulence kinetic energy budget, i.e. its trace vanishes, $\Pi_{i i}=0$. The trace of the Reynolds stress tensor equals $2 k_{t}$, hence in order to arrive at Eq. (5), the relationships $\mathcal{P}_{i i}=2 \mathcal{P}, \epsilon_{i i}=2 \epsilon$ and $\mathcal{D}_{i i}=2 \mathcal{D}$ hold. Further discussion of Reynolds stress models can be found e.g. in Eisfeld ${ }^{26}$ and Al-Sharif. ${ }^{27}$

\section{B. Generalized model equation and resulting transport equation}

We aim to establish a model equation for turbulent velocity from which turbulence kinetic energy and Reynolds stresses derive which are in accordance with those from the $k_{t}$-equation (5) and the model equation (38). To pursue along this line, we seek for a modification to Eq. (35) that directly leads to Eq. (5) and Eq. (38). For this purpose, the following forced linear advection-diffusion-dissipation model equation will be closer studied:

$$
\frac{\partial v_{i}^{\prime}}{\partial t}+v_{j}^{0} \frac{\partial v_{i}^{\prime}}{\partial x_{j}}-\frac{\partial}{\partial x_{j}}\left(D \frac{\partial v_{i}^{\prime}}{\partial x_{j}}\right)+\lambda_{i k} v_{k}^{\prime}=Q_{i},
$$

where

$$
\lambda_{i k}=\lambda \delta_{i k}+f_{i k} .
$$

A spatio-temporal noise term $Q_{i}$ is introduced as a forcing on the right-hand side to effectively model turbulent production. In a precursor step of the derivation of the $k_{t}$-equation from Eq. (39), first we consider the transport equations for the Reynolds stress components. They follow from the application of Eq. (36) together with Eq. (39),

$$
\frac{\partial \mathcal{R}_{i j}}{\partial t}+v_{k}^{0} \frac{\partial \mathcal{R}_{i j}}{\partial x_{k}}=\hat{\mathcal{P}}_{i j}-2 D\left\langle\frac{\partial v_{i}^{\prime}}{\partial x_{k}} \frac{\partial v_{j}^{\prime}}{\partial x_{k}}\right\rangle-2 \lambda \mathcal{R}_{i j}+\frac{\partial}{\partial x_{k}}\left(D \frac{\partial \mathcal{R}_{i j}}{\partial x_{k}}\right)+\Phi_{i j} .
$$

Using Eq. (36), the strength of the resulting production term $\hat{\mathcal{P}}_{i j}$ is proportional to the correlation of the forcing term with the generated fluctuating velocity,

$$
\hat{\mathcal{P}}_{i j}=\left\langle v_{i}^{\prime} Q_{j}+v_{j}^{\prime} Q_{i}\right\rangle .
$$

A new tensor is introduced on the right-hand side of Eq. (41), that is a result of the contractions of tensor $f_{i k}$ from Eq. (39) and the resulting Reynolds stresses, i.e.,

$$
\Phi_{i j}:=f_{i k} \mathcal{R}_{k j}+f_{j k} \mathcal{R}_{k i}
$$


respectively, $\overline{\bar{\Phi}}=\overline{\bar{f}} \overline{\bar{R}}+(\overline{\bar{f}} \overline{\bar{R}})^{T}=\overline{\bar{f}} \overline{\bar{R}}+\overline{\overline{R^{T}}} \overline{\overline{f^{T}}}$. It is easy to proof that $\Phi_{i j}$ reassembles the re-distribution tensor $\Pi_{i j}$ of the Reynolds stress equation (using $\Pi_{i j}=\Pi_{j i}, \overline{\bar{R}}=\overline{\overline{R^{T}}}$, and $\overline{\bar{R}} \overline{\bar{R}}^{-1}=\overline{\bar{I}}$ ) if $f_{i k}$ is defined by

$$
f_{i k}=\frac{1}{2} \Pi_{i j} \mathcal{R}_{j k}^{-1}
$$

where $\mathcal{R}_{j k}^{-1}$ indicates the inverse of the Reynolds stress tensor. In general, $f_{i i} \neq 0$ and $f_{i j} \neq f_{j i}$. However, the resulting tensor satisfies $\Phi_{i j}=\Pi_{i j}$ and in particular $\Phi_{i i}=0$.

Contraction of indices yields the $k_{t}$-equation related to Eq. (39),

$$
\frac{\partial k_{t}}{\partial t}+v_{j}^{0} \frac{\partial k_{t}}{\partial x_{j}}=\hat{\mathcal{P}}-\underbrace{D\left\langle\frac{\partial v_{i}^{\prime}}{\partial x_{j}} \frac{\partial v_{i}^{\prime}}{\partial x_{j}}\right\rangle}_{(I)}-\underbrace{2 \lambda k_{t}}_{(I I)}+\frac{\partial}{\partial x_{j}}\left(D \frac{\partial k_{t}}{\partial x_{j}}\right),
$$

with production term

$$
\hat{\mathcal{P}}=\left\langle v_{i}^{\prime} Q_{i}\right\rangle
$$

Note, since $\Phi_{i i}=\Pi_{i i}=0$, this term does not appear in the $k_{t}$-equation. In principle, the $k_{t}$-equation could be also obtained neglecting $f_{i k}$ in Eq. (39). However, to enforce with Eq. (39) the realization of specific Reynolds stresses, the tensor has to be properly defined. For example, to realize Reynolds stresses in agreement with the Boussinesq assumption Eq. (34), a Reynolds transport model equation (38) has to be formulated in such a way that its Reynolds stresses are equal with those of Eq. (34). This demands for an explicit realization of the re-distribution tensor in the model equation (39).

To be precise, taking the substantial time derivative $D^{0} / D t=\partial / \partial t+v_{j}^{0} \partial / \partial x_{j}$ of Eq. (34) and replacing the substantial time derivative of the turbulence kinetic energy through the transport equation (5) and after rearrangement we arrive at

$$
\frac{\partial \mathcal{R}_{i j}}{\partial t}+v_{k}^{0} \frac{\partial \mathcal{R}_{i j}}{\partial x_{k}}=\underbrace{\frac{2}{3} \delta_{i j} \mathcal{P}}_{=: \mathcal{P}_{i j}}-\underbrace{\frac{\epsilon}{k_{t}} \mathcal{R}_{i j}}_{=: \epsilon_{i j}}+\underbrace{\frac{\partial}{\partial x_{k}}\left(\left(\nu+\frac{\nu_{t}}{\sigma_{k}}\right) \frac{\partial \mathcal{R}_{i j}}{\partial x_{k}}\right)}_{=: \mathcal{D}_{i j}}+\Pi_{i j} .
$$

In this expression dissipation and diffusion terms are obtained by adding and subtracting further terms so that the resulting expressions correspond in form with those realized by Eq. (41). The modeling of the dissipation term $\epsilon_{i j}$ corresponds to the anisotropic dissipation model proposed by Rotta (1951). ${ }^{28}$ The remaining terms lumped together and expressed in terms of the anisotropy tensor $b_{i j}:=\mathcal{R}_{i j}-2 / 3 \delta_{i j} k_{t}=2 \nu_{t} S_{i j}$ provide the definition of the re-distribution tensor needed for the realization of Boussinesq stresses,

$$
\Pi_{i j}=\frac{\epsilon}{k_{t}} b_{i j}-\frac{\partial}{\partial x_{j}}\left(\left(\nu+\frac{\nu_{t}}{\sigma_{k}}\right) \frac{\partial}{\partial x_{j}} b_{i j}\right)+\frac{D^{0}}{D t} b_{i j} .
$$

Since the strain rate tensor is symmetric and satisfies for incompressible flow $S_{i i}=0$, the re-distribution tensor as defined above is symmetric and satisfies $\Pi_{i i}=0$. The term can be computed from any adequate precursor RANS simulation. For stationary problems it is not time dependent. Furthermore, for homogeneous flows it vanishes.

\section{Solenoidal velocity and one-point target statistics}

Eq. (39) is deemed to represent the general extension of Eq. (32) to inhomogeneous flow. Note, even with solenoidal forcing $Q_{i}=\epsilon_{i j k} \partial W_{k} / \partial x_{j}$ non-uniform flow will in general cause a deviation of velocity fluctuations from solenoidality. However, re-distribution and dissipation will enforce the return to homogeneity under a corresponding downstream flow situation.

Weakly non-solenoidal velocity or completely non-solenoidal velocity from non-solenoidal forcing might be acceptable for CAA if velocity fluctuations are not directly applied but are used instead to derive vorticity fluctuations from it that are utilized to prescribe appropriate vortex sound sources, refer to Reiche et al. ${ }^{29}$ for the successful application of vorticity fluctuations from non-solenoidal velocity as source terms in CAA.

Strictly solenoidal velocity fluctuations could be enforced by adding an additional incompressible pressure to the previous transport equation. However this is not considered here, since it realizes a deviation from the 
underlying transport equation of turbulent kinetic energy. The inclusion of an incompressible pressure term in F.L.A.D.D. is in principal possible by solving an additional Poisson equation for pressure. This could be also interesting for general turbulence modeling, as velocity pressure-correlations otherwise are difficult to model. Time-averaged statistics as provided by standard tubulence models can be derived from the unsteady equations using a moving average procedure.

\section{Calibration of model parameter}

\section{Source term calibration variant 1}

To resemble with Eq. (45) the turbulence kinetic energy equation Eq. (5), similarity of the diffusion terms is achieved using

$$
D=\nu+\frac{\nu_{t}}{\sigma_{k}}
$$

There is some degree of freedom in the calibration of production $\hat{\mathcal{P}}$ and inverse time scale $\lambda$ in the model equation. Based on the definition of the diffusion parameter $D$ and that of the dissipation rate Eq. (6), term (I) in Eq. (45) could be substituted by $\epsilon+\frac{\nu_{t}}{\sigma_{k}}\left(\frac{\epsilon}{\nu}\right)$. The first contribution of the two summands realizes the $k_{t}$-dissipation rate $(\epsilon$-term) needed to reassemble Eq. (5). The second contribution would have to be compensated by term $(I I)$, i.e. defining an inverse time scale by

$$
\lambda_{(1)}=-\frac{1}{2 \sigma_{k}} \frac{\nu_{t}}{\nu} \frac{\epsilon}{k_{t}}
$$

where the subscript indicates this specific calibration choice. Thus, a negative damping term in the model equation is obtained. A production term of this kind has been introduced by Stefano \& Vassilyev in the SCALES approach. ${ }^{30}$ The production term then directly defines the forcing via

$$
\hat{\mathcal{P}}_{(1)}=\mathcal{P} .
$$

\section{Source term calibration variant 2}

An alternative is to still use $D$ from Eq. (49) for similarity in the diffusion term but to match the dissipation term $(I I)$ to the pseudo-dissipation, $2 \lambda k_{t}=\epsilon$, hence $\left(\tau_{s}\right.$ from Eq. ( 7$)$ with $c_{\tau}=1$ ),

$$
\lambda_{(2)}=\frac{1}{2} \frac{\epsilon}{k_{t}}=\frac{1}{2 \tau_{s}}
$$

Thus, to provide the $k_{t}$-production $\mathcal{P}$, an effective production term $\hat{\mathcal{P}}$ has to be realized that in addition balances the contribution that comes from dissipation term $(I)$, i.e.

$$
\hat{\mathcal{P}}_{(2)}=\mathcal{P}+\left(2 \lambda_{(2)}+\frac{\nu_{t}}{\sigma_{k}} \chi\right) k_{t}
$$

where $\chi$ denotes an inverse squared length scale. To be precise, $\chi^{-1}=\nu k_{t} / \epsilon=\lambda_{g}^{2} / 10$, where $\lambda_{g}$ is the transverse Taylor length scale. ${ }^{23}$ For this second calibration variant the production would in general not be zero, even if the RANS production term $\mathcal{P}$ vanishes, e.g. for decaying homogenous isotropic turbulence.

Note, to arrive at the relationship Eq. (53), the definition of the pseudo-dissipation

$$
\epsilon=\nu\left\langle\frac{\partial v_{i}^{\prime}}{\partial x_{k}} \frac{\partial v_{i}^{\prime}}{\partial x_{k}}\right\rangle
$$

was utilized. One has to be aware that it is an approximation in the present context, since the right hand side denotes correlations of the generated fluctuating velocity. The $\epsilon$ on the left-hand side is provided by an additional transport equation. Equivalence of both sides implies complete consistency of the turbulence model and full resolution of all velocity fluctuations by F.L.A.D.D. In practice, however, the goal will be the resolution of only the very large-scale part of the velocity fluctuations, so that the right-hand side of Eq. (54) provides only a small fraction of the pseudo-dissipation. The reduction of $\epsilon$ due to the realization of just the large scales can be taken into account by appropriate calibration of function $\chi$ in Eq. (53). For example, 
from scaling $(\epsilon / \nu) \rightarrow c_{\lambda}^{2} k_{t} / \Delta^{2}$, where $\Delta$ denotes the mesh resolution and $c_{\lambda}^{2}$ is a calibration constant, ${ }^{29}$ it derives $\chi \simeq c_{\lambda} / \Delta^{2}$ on a coarse mesh.

The wave-number spectrum that results from the model equation Eq. (39) can be derived for the homogeneous case using the Green function of the linear advection-diffusion-dissipation equation, refer to the next section. It turns out that a positive time scale $\tau_{s}$, Eq. (7) (respectively, inverse time scale $\lambda=\left(2 \tau_{s}\right)^{-1}$ ) is needed to define a turbulence spectrum with plateau and cross-over at wave-number $\sqrt{D / \lambda}$, refer also to the qualitative discussion in Section III. In principle a mixture of variant 1 plus variant 2 with positive time scale would also yield a valid scaling.

However, since the resulting integral length scale that results from scaling variant 2 yields the expected value (as will be shown in the subsequent derivation), the second calibration approach is considered here to be the correct one.

\section{Calibration of production for Reynolds transport equations}

For an extension of the above scaling variant 2 to accomplish a realization of the transport equation Eq. (47) with Eq. (41) the definition of the inverse time-scale Eq. (52) can be used unchanged. The definition of the production tensor depends on the actual assumption made to express the velocity correlation term.

For example, adopting the often made assumption that the dissipation tensor is diagonal, this implies

$$
\epsilon_{i j}=\nu\left\langle\frac{\partial v_{i}^{\prime}}{\partial x_{k}} \frac{\partial v_{j}^{\prime}}{\partial x_{k}}\right\rangle \simeq \frac{2}{3} \delta_{i j} \epsilon
$$

Furthermore, if the production in the Reynolds stress equations is also diagonal, $\mathcal{P}_{i j}=2 / 3 \delta_{i j} \mathcal{P}$, the gross production tensor to be realized by the F.L.A.D.D. forcing eventually is diagonal as well, viz $\hat{\mathcal{P}}_{i j}=\frac{2}{3} \delta_{i j} \hat{\mathcal{P}}$, with $\hat{\mathcal{P}}$ from Eq. (53). For more general cases with anisotropic Reynolds stress production and anisotropic dissipation described by the model of Rotta, ${ }^{28} \epsilon_{i j}=\epsilon / k_{t} \mathcal{R}_{i j}$, implies realization of a non-diagonal gross production tensor,

$$
\hat{\mathcal{P}}_{i j}=\mathcal{P}_{i j}+\left(2 \lambda+\frac{\nu_{t}}{\sigma_{k}} \chi\right) \mathcal{R}_{i j}
$$

Alternatively, based on the definition of an anisotropic length-scale function ${ }^{\mathrm{d}} \chi_{i j}:=2\left\langle\frac{\partial v_{i}^{\prime}}{\partial x_{k}} \frac{\partial v_{j}^{\prime}}{\partial x_{k}}\right\rangle /\left\langle v_{l}^{\prime} v_{l}^{\prime}\right\rangle$, an anisotropic production follows from

$$
\hat{\mathcal{P}}_{i j}=\mathcal{P}_{i j}+2\left(\nu+\frac{\nu_{t}}{\sigma_{k}}\right) \chi_{i j} k_{t}
$$

\section{E. Green function of the linear advection-diffusion-dissipation equation}

\section{General discussion}

In order to provide the stochastic F.L.A.D.D. forcing by an adapted FRPM procedure, as a prerequisite the variance of the forcing term has to be derived based on the value of the gross production term as specified in the previous paragraphs. Furthermore, for homogeneous isotropic turbulence the meaningfulness of the generated turbulence spectrum must be verified. To tackle these questions the Green function of the linear equations solved is utilized.

It is convenient to consider solutions in a Lagrangian frame, for which the advective term explicitly drops out and the governing equation reduces to an inhomogeneous diffusion-dissipation equation

$$
\frac{\partial v_{i}^{\prime}}{\partial t}-\frac{\partial}{\partial x_{j}}\left(D \frac{\partial v_{i}^{\prime}}{\partial x_{j}}\right)+\lambda_{i j} v_{j}^{\prime}=Q_{i}
$$

where $\lambda_{i j}:=\lambda \delta_{i j}+f_{i j}$. For solenoidal forcing (SF)

$$
Q_{i}=\epsilon_{i j k} \frac{\partial W_{j}}{\partial x_{k}},
$$

\footnotetext{
${ }^{\mathrm{d}}$ The tensor $\chi_{i j}$ may result also from a moving average technique.
} 
for non-solenoidal forcing (NSF)

$$
Q_{i}=W_{i}
$$

The derivation of analytical solutions for the model equations benefits from specific case dependent possible simplifications:

1. The resulting wave-number and frequency spectra and a proper calibration of the forcing spectrum have to be derived from two-points statistics for homogeneous isotropic turbulence.

2. For the more general case of inhomogeneous turbulence just the proper variance of the white-noise forcing term (one-point statistics) must be specified to accomplish the desired local target turbulence production rate.

\section{Green function for wave-number spectra in homogeneous flow}

In the first homogeneous case discussed above two-point cross-correlations of type $C(\boldsymbol{r}, \tau)=\left\langle v_{i}(\boldsymbol{x}, t) v_{j}(\boldsymbol{x}+\boldsymbol{r}, t+\tau)\right.$ have to be evaluated. Homogeneity of the problem means to use $D=$ const and $\lambda_{i j} \rightarrow \lambda \delta_{i j}$ in Eq. (58). Hence, all equations decouple and it is sufficient to consider each equation individually, i.e. just studying the scalar equation

$$
\frac{\partial v^{\prime}}{\partial t}-D \frac{\partial^{2} v^{\prime}}{\partial x_{j}^{2}}+\lambda v=Q
$$

The free-space solution to Eq. (61) formally reads

$$
v^{\prime}(\boldsymbol{x}, t)=\int_{-\infty}^{\infty} \int_{-\infty}^{\infty} \mathcal{G}\left(\boldsymbol{x}, t, \boldsymbol{x}^{\prime}, t^{\prime}\right) Q\left(\boldsymbol{x}^{\prime}, t^{\prime}\right) \mathrm{d}^{n} \boldsymbol{x}^{\prime} \mathrm{d} t^{\prime},
$$

where $\mathcal{G}$ denotes the causal free-space Green function of the diffusion-dissipation equation Eq. (61), which has to satisfy

$$
\mathcal{L G}\left(\boldsymbol{x}, t, \boldsymbol{x}^{\prime}, t^{\prime}\right)=\delta\left(\boldsymbol{x}-\boldsymbol{x}^{\prime}\right) \delta\left(t-t^{\prime}\right),
$$

with operator $\mathcal{L}:=\left\{\frac{\partial}{\partial t}-D \frac{\partial^{2}}{\partial x_{j}^{2}}+\lambda\right\}$. As discussed elsewhere, the Green function explicitly derives as

$$
\mathcal{G}\left(\boldsymbol{x}, t, \boldsymbol{x}^{\prime}, t^{\prime}\right)=\frac{H\left(t-t^{\prime}\right) \exp \left(-\lambda\left(t-t^{\prime}\right)\right)}{\sqrt{\left(4 \pi D\left(t-t^{\prime}\right)\right)^{n}}} \exp \left(-\frac{\left|\boldsymbol{x}-\boldsymbol{x}^{\prime}\right|^{2}}{4 D\left(t-t^{\prime}\right)}\right),
$$

where $H\left(t-t^{\prime}\right)$ denotes a Heaviside function and $n$ indicates the dimension of the problem. It is worth to note that for vanishing time difference $\left(t-t^{\prime}\right) \rightarrow 0^{+}$, the spatial dependency of the previous Green function becomes a delta function, i.e.,

$$
\lim _{\left(t-t^{\prime}\right) \rightarrow 0^{+}} \mathcal{G}\left(\boldsymbol{x}, t, \boldsymbol{x}^{\prime}, t^{\prime}\right) \rightarrow \delta\left(\boldsymbol{x}-\boldsymbol{x}^{\prime}\right)
$$

\section{Green function for calibration of forcing}

In the second case, the production is defined by $\mathcal{P}_{i j}(\boldsymbol{x}, t)=\left\langle v_{i}^{\prime}(\boldsymbol{x}, t) Q_{j}(\boldsymbol{x}, t)+v_{j}^{\prime}(\boldsymbol{x}, t) Q_{i}(\boldsymbol{x}, t)\right\rangle$ and the one-point correlations have to be evaluated. A causal free-space matrix Green function for the solution of Eq. (58) with constant diffusion and dissipation tensor can be derived. By introducing the operator $\mathcal{L}_{i j}:=\left\{\delta_{i j}\left(\frac{\partial}{\partial t}-D \frac{\partial^{2}}{\partial x_{k}^{2}}\right)+\lambda_{i j}\right\}$, the equation to be solved can be written

$$
\mathcal{L}_{i j} v_{j}^{\prime}=Q_{i} .
$$

The causal free-space matrix Green function is defined by

$$
\mathcal{L}_{i j} \mathcal{G}_{j k}:=\delta_{i k} \delta\left(\boldsymbol{x}-\boldsymbol{x}^{\prime}\right) \delta\left(t-t^{\prime}\right),
$$

from which the solution derives via

$$
v_{j}^{\prime}=\mathcal{G}_{j k} \star Q_{k}=\int \mathcal{G}_{j k}\left(\boldsymbol{x}-\boldsymbol{x}^{\prime}, t-t^{\prime}\right) Q_{k}\left(\boldsymbol{x}^{\prime}, t^{\prime}\right) \mathrm{d}^{3} \boldsymbol{x}^{\prime} \mathrm{d} t .
$$


As discussed elsewhere, the matrix Green function reads

$$
\mathcal{G}_{j k}\left(\boldsymbol{x}, t, \boldsymbol{x}^{\prime}, t^{\prime}\right)=\frac{H\left(t-t^{\prime}\right) \exp _{j k}\left(-\overline{\bar{\lambda}}\left(t-t^{\prime}\right)\right)}{\sqrt{\left(4 \pi D\left(t-t^{\prime}\right)\right)^{n}}} \exp \left(-\frac{\left|\boldsymbol{x}-\boldsymbol{x}^{\prime}\right|^{2}}{4 D\left(t-t^{\prime}\right)}\right)
$$

where $\exp _{j k}$ denotes the matrix exponential ${ }^{31}$ and $\overline{\bar{\lambda}}$ denotes the matrix corresponding to components $\lambda_{i j}$. The first order approximation to the matrix exponential valid for small arguments of $t-t^{\prime}$ reads

$$
\exp _{i k}\left(-\overline{\bar{\lambda}}\left(t-t^{\prime}\right)\right) \simeq \delta_{i k}-\lambda_{i k}\left(t-t^{\prime}\right)+\cdots .
$$

Hence, for vanishing time difference $\left(t-t^{\prime}\right) \rightarrow 0^{+}$, the spatial dependency of the previous Green function becomes a delta function as well, i.e.,

$$
\lim _{\left(t-t^{\prime}\right) \rightarrow 0^{+}} \mathcal{G}_{i k}\left(\boldsymbol{x}, t, \boldsymbol{x}^{\prime}, t^{\prime}\right) \rightarrow \delta_{i k} \delta\left(\boldsymbol{x}-\boldsymbol{x}^{\prime}\right) .
$$

Without further proof given here, the previous feature, i.e. that the Green function reduces in the limit of vanishing time difference to a delta function, also holds for spatially variable diffusion and dissipation tensors.

\section{F. Calibration of forcing}

A non-solenoidal source term similar to the one defined by Eq. (23) reads

$$
Q_{i}(\boldsymbol{x}, t)=\int G\left(\left|\boldsymbol{x}-\boldsymbol{x}^{\prime}\right|, l\right) a_{i j}\left(\boldsymbol{x}^{\prime}, t\right) \xi_{j}\left(\boldsymbol{x}^{\prime}, t\right) \mathrm{d}^{n} \boldsymbol{x}^{\prime}
$$

where $G=(\sqrt{2 \pi} l)^{-n} \exp \left(-\frac{r^{2}}{2 l^{2}}\right)$ is a Gaussian normal distribution of length scale $l$ and $n \in\{2,3\}$ is the subsequently considered dimension of the problem. The tensor $a_{i j}$ has to be specified to accomplish proper production. This source can be generated with FRPM, e.g. compare it to Eq. (10) and the discussion there.

Using the matrix Green function of the previous paragraph to express the generated velocity in terms of its convolution with the source vector, the generic production term $\hat{\mathcal{P}}_{i j}^{*}(\boldsymbol{x}, t)=\left\langle v_{i}^{\prime}(\boldsymbol{x}, t) Q_{j}(\boldsymbol{x}, t)\right\rangle$ becomes (for brevity possible time dependence of $\hat{\mathcal{P}}_{i j}(\boldsymbol{x}, t)$ and $a_{i j}(\boldsymbol{x}, t)$ and dependence of $G$ on $l$ not shown here)

$\hat{\mathcal{P}}_{i j}^{*}(\boldsymbol{x})=\iint_{\boldsymbol{x}^{\prime}} \int_{\boldsymbol{x}^{\prime \prime}} \int_{\boldsymbol{x}^{\prime \prime \prime}} \mathcal{G}_{i k}\left(\boldsymbol{x}, t, \boldsymbol{x}^{\prime}, t^{\prime}\right) G\left(\boldsymbol{x}^{\prime}-\boldsymbol{x}^{\prime \prime}\right) G\left(\boldsymbol{x}-\boldsymbol{x}^{\prime \prime \prime}\right) a_{k l}\left(\boldsymbol{x}^{\prime \prime}\right) a_{j m}\left(\boldsymbol{x}^{\prime \prime \prime}\right)\left\langle\xi_{l}\left(\boldsymbol{x}^{\prime \prime}, t^{\prime}\right) \xi_{m}\left(\boldsymbol{x}^{\prime \prime \prime}, t\right)\right\rangle \mathrm{d}^{n} \boldsymbol{x}^{\prime} \mathrm{d}^{n} \boldsymbol{x}^{\prime \prime} \mathrm{d}^{n} \boldsymbol{x}^{\prime \prime \prime} \mathrm{d} t^{\prime}$.

Similar to Eqs. (14) and (15), the source term is defined by

$$
\left\langle\xi_{l}\left(\boldsymbol{x}^{\prime \prime}, t^{\prime}\right)\right\rangle=0, \quad\left\langle\xi_{l}\left(\boldsymbol{x}^{\prime \prime}, t^{\prime}\right) \xi_{m}\left(\boldsymbol{x}^{\prime \prime \prime}, t\right)\right\rangle=\delta_{l m} \delta\left(t^{\prime}-t\right) \delta\left(\boldsymbol{x}^{\prime \prime \prime}-\boldsymbol{x}^{\prime \prime}\right),
$$

so that integrations with respect to $\boldsymbol{x}^{\prime \prime \prime}$ and $t^{\prime}$ can be carried out immediately, yielding

$$
\hat{\mathcal{P}}_{i j}^{*}(\boldsymbol{x}, t)=\int_{\boldsymbol{x}^{\prime}} \int_{\boldsymbol{x}^{\prime \prime}} \underbrace{\mathcal{G}_{i k}\left(\boldsymbol{x}, t, \boldsymbol{x}^{\prime}, t^{\prime} \rightarrow t\right)}_{\delta_{i k} \delta\left(\boldsymbol{x}-\boldsymbol{x}^{\prime}\right)} G\left(\boldsymbol{x}^{\prime}-\boldsymbol{x}^{\prime \prime}, l\right) G\left(\boldsymbol{x}-\boldsymbol{x}^{\prime \prime}, l\right) c_{k j}\left(\boldsymbol{x}^{\prime \prime}, t\right) \mathrm{d}^{n} \boldsymbol{x}^{\prime} \mathrm{d}^{n} \boldsymbol{x}^{\prime \prime} .
$$

Here the symmetric tensor $c_{k j}=a_{k l} a_{j l}$ is introduced. As indicated, in the limit $t^{\prime} \rightarrow t$ the matrix Green function reduces to a delta function, refer to the previous section. Hence, integration with respect to $\boldsymbol{x}^{\prime}$ can be carried out, yielding

$$
\hat{\mathcal{P}}_{i j}^{*}(\boldsymbol{x}, t)=\int_{\boldsymbol{x}^{\prime}} G^{2}\left(\boldsymbol{x}-\boldsymbol{x}^{\prime \prime}, l\right) c_{i j}\left(\boldsymbol{x}^{\prime \prime}, t\right) \mathrm{d}^{n} \boldsymbol{x}^{\prime \prime} .
$$

The forcing length scale usually is small, as it limits the resolved largest wave-numbers to the maximal mesh resolution. The actual turbulent length scale unfolds as an effect of the diffusion process solved. For small length scales, the mean value theorem allows to place the variance scaling function $c_{i j}\left(\boldsymbol{x}^{\prime \prime}, t\right)$ outside the integral, evaluated approximately at position $\boldsymbol{x}$. The integration of the squared Gaussian can be carried 
out, yielding $c_{i j}=\hat{\mathcal{P}}_{i j}^{*}$. The complete production tensor is defined by Eq. (4), i.e. $\hat{\mathcal{P}}_{i j}=\hat{\mathcal{P}}_{i j}^{*}+\hat{\mathcal{P}}_{j i}^{*}$, hence eventually it follows

$$
c_{i j}(\boldsymbol{x}, t)=\frac{1}{2} \hat{\mathcal{P}}_{i j}(\boldsymbol{x}, t)
$$

The previous expression is the main result of this section. From tensor $c_{i j}$, the tensor $a_{i j}$ needed in Eq. (72) derives via Cholesky decomposition, refer to the discussion in Reiche et al. ${ }^{29}$

Scaling laws for solenoidal forcing derive accordingly. Since the equations solved are linear, a superposition of sources is possible, allowing to derive in addition in wave-number space forcing terms with prescribed production. Without proof given here, the previous result also holds for the general case of variable diffusion.

\section{G. Resulting wave-number spectra and spatial correlation functions}

\section{3-D non-solenoidal forcing}

To compute the resulting wave-number and frequency spectra for homogeneous flow the velocity correlation tensor is derived in a first step from the corresponding Green function, Eq. (64). To simplify the analysis, the cut-off length scale of the Gaussian in Eq. (72) is taken to be $l \rightarrow 0$, so that $Q=a \xi$ results (only one component considered, i.e. $\xi_{i} \rightarrow \xi, a_{i j} \rightarrow a, c_{i j} \rightarrow c=a^{2}$ ). By expressing the velocity field in terms of the convolution of the source term with the Green function the cross-correlation function follows from

$$
\begin{aligned}
\mathcal{R}(\boldsymbol{r}, \tau) & :=\langle v(\boldsymbol{x}, t) v(\boldsymbol{x}+\boldsymbol{r}, t+\tau)\rangle \\
= & \int_{\boldsymbol{x}^{\prime}} \int_{\boldsymbol{x}^{\prime \prime}} \int_{t^{\prime}} \int_{t^{\prime \prime}} \mathcal{G}\left(\boldsymbol{x}, t, \boldsymbol{x}^{\prime}, t^{\prime}\right) \mathcal{G}\left(\boldsymbol{x}+\boldsymbol{r}, t+\tau, \boldsymbol{x}^{\prime \prime}, t^{\prime \prime}\right)\left\langle Q\left(\boldsymbol{x}^{\prime}, t^{\prime}\right) Q\left(\boldsymbol{x}^{\prime \prime}, t^{\prime \prime}\right)\right\rangle \mathrm{d}^{n} \boldsymbol{x}^{\prime} \mathrm{d}{ }^{n} \boldsymbol{x}^{\prime \prime} \mathrm{d} t^{\prime} \mathrm{d} t^{\prime \prime} .
\end{aligned}
$$

The source term adopts the properties of $\xi$, i.e. $\left\langle Q\left(\boldsymbol{x}^{\prime}, t^{\prime}\right) Q\left(\boldsymbol{x}^{\prime \prime}, t^{\prime \prime}\right)\right\rangle=c \delta\left(t^{\prime \prime}-t^{\prime}\right) \delta\left(\boldsymbol{x}^{\prime \prime}-\boldsymbol{x}^{\prime}\right)$. Carrying out the integration with respect to $t^{\prime \prime}$ and $\boldsymbol{x}^{\prime \prime}$ using the Green function $\mathcal{G}\left(\boldsymbol{x}-\boldsymbol{x}^{\prime}, t-t^{\prime}\right)$ as defined by Eq. (64) and making the substitution $\boldsymbol{u}=-\left(\boldsymbol{x}-\boldsymbol{x}^{\prime}\right)$, together with the symmetry of the Green function it follows

$$
\mathcal{R}(\boldsymbol{r}, \tau)=c \int_{\boldsymbol{x}^{\prime}} \int_{t^{\prime}} \mathcal{G}\left(\boldsymbol{u}, t-t^{\prime}\right) \mathcal{G}\left(\boldsymbol{r}-\boldsymbol{u}, t+\tau-t^{\prime}\right) \mathrm{d}^{n} \boldsymbol{x}^{\prime} \mathrm{d} t^{\prime} .
$$

Using further the Fourier wave-number transform $\hat{\mathcal{G}}$ of $\mathcal{G}$, the spatial convolution in the previous expression is rewritten in terms of the product of its wave-number transform. Carrying out the time integration we arrive at

$$
\hat{\mathcal{R}}(\boldsymbol{k}, \tau) \propto \frac{\exp \left(-\left(\lambda+k^{2} D\right)|\tau|\right)}{1+k^{2} l_{s}^{2}}
$$

with a characteristic length scale defined by

$$
l_{s}^{2}:=\frac{D}{\lambda}
$$

and $k=|\boldsymbol{k}|$. Back transformation from 3-D wave-number space into space yields the cross-correlation function in a moving frame of reference $\mathcal{R}(\boldsymbol{r}, \tau)$. The cross-correlation function in a fixed frame of reference $\mathcal{R}^{*}$ results replacing $\boldsymbol{r} \rightarrow \boldsymbol{r}-\boldsymbol{v}^{0} \tau$, i.e. $\mathcal{R}^{*}(\boldsymbol{r}, \tau)=\mathcal{R}\left(\boldsymbol{r}-\boldsymbol{v}^{0} \tau, \tau\right)$. Hence, for $\tau=0$ the moving frame cross-correlation function corresponds to the fixed frame, $\mathcal{R}^{*}(\boldsymbol{r}, 0)=\mathcal{R}(\boldsymbol{r}, 0)$. Back transformation of the more general expression

$$
\hat{C}(\boldsymbol{k})=\frac{A}{\left(1+k^{2} l_{s}^{2}\right)^{3 / 2+\nu}},
$$

which corresponds to Eq. (79) for $\tau=0$ and $\nu=-1 / 2$, yields

$$
C(r) \propto\left(r / l_{s}\right)^{\nu} K_{\nu}\left(r / l_{s}\right),
$$

i.e. is a spherical function just depending on $r=|\boldsymbol{r}|$ (in the above expression $K_{\alpha}$ denotes the modified Bessel function of second kind and order $\alpha$ ). The one-dimensional correlation function $f\left(x_{1}\right)$ for non-solenoidal forcing follows from expression Eq. (82) setting $x_{1}=r, x_{2}=x_{3}=0$, i.e., $f\left(x_{1}\right)=C\left(x_{1}\right)$. 


\section{3-D solenoidal forcing}

Considering 3-D solenoidal forcing, for homogeneous flow Eq. (58) is rewritten

$$
\frac{\partial q_{i}}{\partial t}-D \frac{\partial^{2} q_{i}}{\partial x_{j}^{2}}+\lambda q_{i}=W_{i}
$$

using $Q_{i}=\epsilon_{i j k} \partial W_{k} / \partial x_{j}, \lambda_{i j}=\lambda \delta_{i j}$, and $v_{i}^{\prime}:=\epsilon_{i j k} \partial q_{k} / \partial x_{j}$. For mutually uncorrelated components $W_{i}$ each component equation is solved independently with the Green function Eq. (64).

In particular, the solution procedure of the non-solenoidal case discussed before is valid for each component of the vector potential, thus we arrive at

$$
\mathcal{R}_{i j}(\boldsymbol{r}, 0)=\left\langle q_{i}(\boldsymbol{x}, t) q_{j}(\boldsymbol{x}+\boldsymbol{r}, t)\right\rangle=\delta_{i j} C(r),
$$

with $C(r)$ defined by Eq. (82). It was discussed in Ewert ${ }^{7}$ that velocity fluctuations from the curl of $q_{i}$ realize the cross-correlation tensor of homogeneous-isotropic turbulence. ${ }^{32}$ In particular, referring to Ref. ${ }^{7}$ the non-normalized longitudinal correlation function that results is given by

$$
f(r)=-\frac{C^{\prime}(r)}{r}
$$

where the dash indicates differentiation with respect to $r$. Starting from expression

$$
\hat{C}(\boldsymbol{k})=\frac{A}{\left(1+k^{2} l_{s}^{2}\right)^{5 / 2+\nu}},
$$

instead of Eq. (81), its back transformation yields a function $C(r)$ equal to Eq. (82) with $\nu$ replaced by $\nu+1$. Differentiation of the result according to Eq. (85) and exploiting some properties of the modified Bessel function, the longitudinal correlation function follows to be given by

$$
f(r) \propto\left(r / l_{s}\right)^{\nu} K_{\nu}\left(r / l_{s}\right) .
$$

To conclude, to arrive at the same longitudinal correlation function Eq. (87), for solenoidal forcing the power of the denominator Eq. (86) has to be increased by one compared to that of the denominator Eq. (81) needed for non-solenoidal forcing. In 2-D, to arrive at the longitudinal correlation function Eq. (87) the denominator powers are $1+\nu$ and $2+\nu$ for non-solenoidal and solenoidal forcing, respectively.

\section{One-dimensional wave-number spectrum from 3-D forcing}

One-dimensional wave-number transformation of the longitudinal correlation function Eq. (87) yields the corresponding one-dimensional wave-number spectrum ${ }^{23}$

$$
\hat{f}\left(k_{1}\right) \propto\left(1+k_{1}^{2} l_{s}^{2}\right)^{-\frac{1+2 \nu}{2}},
$$

i.e. a low wave-number plateau, followed by decay $\propto k_{1}^{-(1+2 \nu)}$. For $\nu=1 / 2$ a decay with power -2 results, i.e. a one-dimensional Liepmann spectrum. For $\nu=1 / 3$ a decay with exponent $-5 / 3$ would result.

For advection dominated problems application of Taylor's hypothesis for a constant (homogeneous) advection velocity $v_{1}^{0}$ in $x_{1}$-direction yields an approximation to the resulting frequency spectrum,

$$
\Phi(\omega) \simeq \hat{f}\left(\omega / v_{1}^{0}\right) .
$$

Formally, the exact frequency spectrum follows from the frequency Fourier transform $\Phi(\omega) \bullet \multimap \mathcal{R}^{*}(0, \tau)$. However, for 'slow' decay Eq. (89) might realize an excellent expression for the latter result. Fig. 6 shows a comparison of both frequency spectra and simulation results. The parameter defining the slowness of decay is given by

$$
\alpha=v_{1}^{0} / l_{s} \lambda .
$$

For $\alpha \rightarrow \infty$ it is frozen turbulence, i.e. Taylor's hypothesis is exactly valid. In Fig. $6 \alpha=10$ is used. The additional effect of turbulent decay causes slight deviations of the spectrum from Taylor's hypothesis with an exponent smaller than 2 .

\section{Colored forcing}


The previous results indicate that an exponent 2 is needed in the denominator of Eq. (81) for the generation of Liepmann spectra from non-solenoidal 3 -D forcing. For solenoidal forcing it is 3. A genuine non-solenoidal point forcing yields exponent 1 in the actual denominator, refer to Eq. (79), which can be enhanced with 'extra powers' in two different ways. First, as discussed in the appendix, a colored forcing can be applied, realizing the source $W_{i}$ by the weighted sum of mutually uncorrelated source terms of different length-scales. The wave-number spectum realized by the forcing eventually appears as a multiplier of Eq. (79). Effectively, to achieve a Liepmann spectrum from solenoidal forcing the source term $W_{i}$ has to realize wave-number spectrum

$$
S(\boldsymbol{k}) \propto \frac{l_{s}^{2} k^{2}}{\left(1+k^{2} l_{s}^{2}\right)^{2}} .
$$

However, this procedure of reintroducing an isotropic model for the forcing term may be seen to have the conceptual disadvantages that the two-point statistics do not entirely unfold from simple point like forcing.

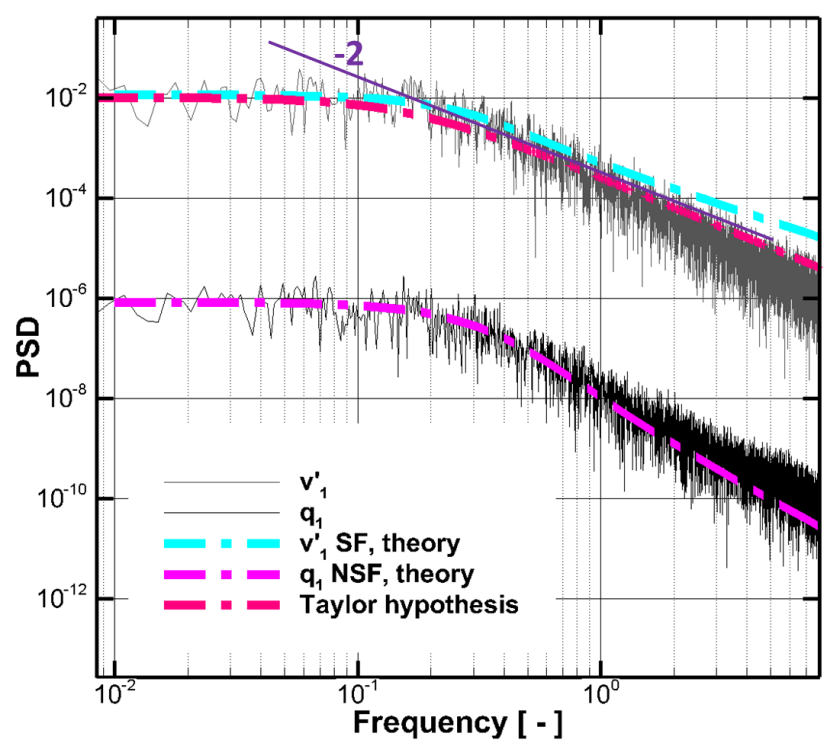

Figure 6. Frequency spectrum from Eq. (89) based on Taylor hypothesis and theoretical frequency spectra (theory) in relation to simulation results, 3-D non-solenoidal and solenoidal forcing (2-step approach), $D=0.1, \lambda=0.1$, $v_{1}^{0}=1.0, l=0.007$.

Alternatively, an expression equal to Eq. (87) with $\nu=1 / 2$ (exponent 3) results from a consecutively solved additional set of F.L.A.D.D. equations, where the output of the first set serves as input for the second set. The exponent result follows from the procedure sketched in G.1 by replacing the Green function with its self-convolution. Using solenoidal point forcing, eventually a decay law of Liepmann characteristic with exponent '-2' follows for the resulting wave-number spectrum. Fig. 6 shows simulation results for this 2 -step approach. The numerical obtained slope is slightly below the expected trend. The generation of colored noise from a doubled set of Langevin equations without diffusion term was discussed in Refs. 33 and 19.

\section{Resulting integral length scale}

The effective resulting integral length scale from application of a doubled set of equations theoretical yields $\nu=1 / 2$. For this value, the normalized longitudinal correlation function from Eq. (87) yields $f(r)=$ $\exp \left(-r / l_{s}\right),{ }^{23}$ with length scale from Eq. (80). As a result, for $\nu=1 / 2$ the resulting integral length scale equals $l_{s}$. If $\lambda$ and $D$ are derived from RANS scaling variant 2, Eq. (52), the length scale infers from

$$
l_{s}=\sqrt{2\left(\nu+\frac{\nu_{t}}{\sigma_{k}}\right) \tau_{s}} \simeq \sqrt{\frac{2}{\sigma_{k}}} \sqrt{\nu_{t} \tau_{s}}=c_{l} \frac{k_{t}^{3 / 2}}{\epsilon}, \quad c_{l}=\sqrt{\frac{2 c_{\tau} C_{\mu}}{\sigma_{k}}},
$$

with $\tau_{s}$ and $\nu_{t}$ defined by Eqs. (7) and (8), i.e. the result already anticipated in Section III. The result shows that an explicit dissipation term $\lambda$ with positive time constant $\tau_{s}$ is needed to realize the proper shape of the resulting turbulence spectrum. For values $c_{\tau}=1.0, C_{\mu}=0.09$, and $\sigma_{k}=1.0$ (from $k_{t}-\omega$ model setting), the result is $c_{l}=0.424$, i.e. close to the asymptotic value $c_{l}=0.43$ discussed in Pope. ${ }^{23}$

\section{H. Turbulent energy cascade}

The previous results indicate that a high wave-number forcing (production) at small length scale produces spectra with larger integral scale, i.e. seem to imply that a shift of energy occurs from larger to smaller wave-numbers, which is opposite to the energy cascade expected for real turbulence. However, based on the scaling variant 2 discussed above, the energy cascade is maintained as follows: in equilibrium the dissipation term provides a broadband lowering of energy in the wave-number domain, which is compensated by a production of energy in the high wave-numbers, i.e. effectively a shift of the energy from lower to higher wave-numbers. Fig. 7 depicts the energy transfer function realized by F.L.A.D.D., showing a reduction for 
lower wave-numbers in favor of an increase in the high wave-numbers. The qualitative shape is in very good qualitative agreement with results from Direct Numerical Simulation, compare to Figure 6.2 in Lesieur. ${ }^{34}$ Details related to the definition of the transfer function can be also found there.

\section{Numerical Method}

For numerical test solutions of the F.L.A.D.D. a simple second order spatial discretization is used for the diffusion term. Since the source term is defined in a Lagrangian frame and for stationary problems a constant mean-flow is present, application of an Arbitrary Lagrangian-Eulerian (ALE) scheme $^{35}$ is advantageous.

That is, the equation system is transformed via Galilean transformation into a Lagrangian frame. Hence, the convective term is simply tranformed away (refer to the discussion in Section V.E.1).

After finalization of one time step, the solution is given on a modified mesh defined by the position of the mesh points advected in the mean flow from their initial positions at time level $n$ to a new positions at time level $n+1$, where the displacement is determined by the time step size $\Delta t$.

Since a two-step time advancement is used, besides the regular initial mesh there is need for one additional mesh defined by the displaced mesh points at the next higher time level. In a remapping

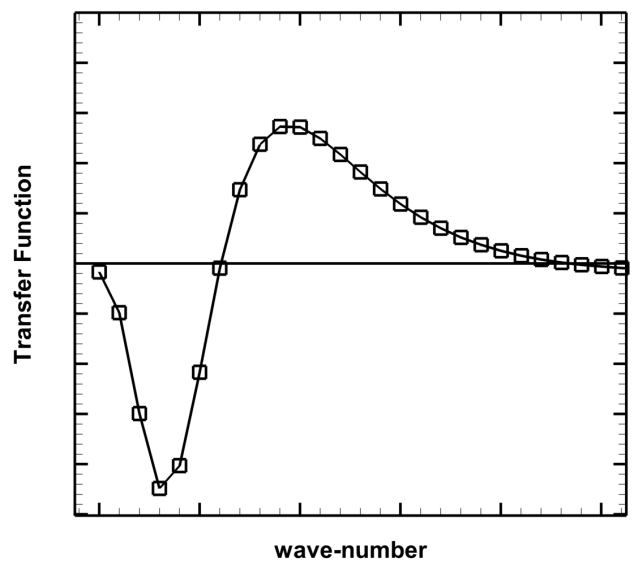
step, the solution at the new time level is interpolated from the second mesh back onto the initial mesh. Since the mean-flow is stationary, the second mesh and the remapping coefficients are fixed and determined once at the start of a computation.

The discretized linear advection-diffusion-dissipation equation in the Lagrangian frame reads

$$
\frac{\mathrm{d} \boldsymbol{v}}{\mathrm{d} t}=\boldsymbol{A}(t) \boldsymbol{v}+\boldsymbol{B}(t) \boldsymbol{r}(t)
$$

Discussing the 3-D implementation (for 2-D it is equivalent), $\boldsymbol{v}$ is a $3 N$-component vector defined by all three velocity components $\left(v_{1}^{\prime}, v_{2}^{\prime}, v_{3}^{\prime}\right)_{j}$ for each of the $j \in\{1, \ldots, N\}$ points of the Lagrangian mesh in use. The matrix $\boldsymbol{A}(t)$ describes the discretization of the diffusion and dissipation terms. It includes besides the coupling of different components through the re-distribution tensor and the spatial dependence of the diffusion constant and inverse time scale also the mutual coupling of different mesh points due to the spatial discretization method applied.

Furthermore, $\boldsymbol{r}(t)$ is a forcing vector that formally results from a FRPM discretization of the source Eg. (72) similar to Eqs. (16) and (17),

$$
Q_{i}\left(\boldsymbol{x}_{j}, t\right) \simeq \sum_{p} G\left(\boldsymbol{x}_{j}, \boldsymbol{x}_{p}\right) a_{i j}\left(\boldsymbol{x}_{p}, t\right) \sqrt{\delta V_{p}} r_{i p}(t), \quad\left\langle r_{i p}(t)\right\rangle=0, \quad\left\langle r_{i p}(t) r_{j k}\left(t^{\prime}\right)\right\rangle=\delta_{i j} \delta_{p k} \delta\left(t-t^{\prime}\right),
$$

where the index $p, k$ defines the particle number of the $P$ particles involved and the source vector has $3 \times P$ components $\boldsymbol{r}=\left(\ldots, r_{1 p}, r_{2 p}, r_{3 p}, \ldots\right)$. Furthermore, $\boldsymbol{x}_{p}$ is the particle position. The proper scaling of tensor $a_{i j}$ was derived in Section V.F. Note, the length of $\boldsymbol{r}$ could be different from that of $\boldsymbol{v}$. The matrix $\boldsymbol{B}$ comprises all FRPM filter coefficients and the forcing amplitudes from Eq. (94). As indicated, in general both matrices $\boldsymbol{A}, \boldsymbol{B}$ could exhibit a time dependence in the Lagrangian frame from inhomogeneous diffusion, dissipation, and production. The matrices are sparse, e.g. second order structured discretization of the diffusion term typically involves 9 nodes. The white-noise source term Eq. (94) is deemed to result from a non-white noise term in the limit of vanishing correlation time.

Starting from Eq. (93) with a non-white noise source term, the linear Stratonovich stochastic differential 
equation with additive (commutative) noise results in this limit, ${ }^{36}$

$$
\mathrm{d} \boldsymbol{v}=\boldsymbol{A}(t) \boldsymbol{v} \mathrm{d} t+\boldsymbol{B}(t) \mathrm{d} \boldsymbol{W}(t) .
$$

The notation $\mathrm{d} \boldsymbol{W}(t)$ explicitly indicates that the noise source is replaced by a stochastic delta correlated Markov process. For time integration an implicit second order Euler method (IE-2) is used. The implicit IE-2 procedure corresponds to the 'strong vector semi-implicit algorithm' discussed in section 10.5.5 of the monograph of Gardiner. ${ }^{36}$ Exploiting the fact that $\boldsymbol{B}$ does not depend on $\boldsymbol{v}$, the strong vector semi-implicit algorithm reads

$$
\begin{aligned}
& \overline{\boldsymbol{v}}^{n}=\frac{1}{2}\left(\boldsymbol{v}^{n+1}+\boldsymbol{v}^{n}\right), \quad \bar{t}_{n}=\frac{1}{2}\left(t_{n+1}+t_{n}\right), \\
& \overline{\boldsymbol{A}}^{n}=\boldsymbol{A}\left(\bar{t}_{n}\right) \simeq \frac{1}{2}\left(\boldsymbol{A}\left(t_{n+1}\right)+\boldsymbol{A}\left(t_{n}\right)\right), \quad \overline{\boldsymbol{B}}^{n}=\boldsymbol{B}\left(\bar{t}_{n}\right) \simeq \frac{1}{2}\left(\boldsymbol{B}\left(t_{n+1}\right)+\boldsymbol{B}\left(t_{n}\right)\right) \\
& \boldsymbol{v}^{n+1}=\boldsymbol{v}^{n}+\overline{\boldsymbol{A}}^{n} \overline{\boldsymbol{v}}^{n} \Delta t+\overline{\boldsymbol{B}}^{n} \Delta \boldsymbol{W}^{n},
\end{aligned}
$$

where $\Delta t=t_{n+1}-t_{n}$. Here, $\Delta \boldsymbol{W}^{n}$ represents a Wiener process (a specific Markov process), realized for each random particle. That is, $\Delta W_{i k}^{n}=\sqrt{\Delta t} \sigma_{i k}^{n}$, with $\sigma_{i k}^{n}$ defined by Eq. (19). The semi-implicit algorithm is A-stable and has both strong and weak order of convergence $\Delta t^{1}$. The numerical solution was verified with the analytical solution of the Langevin equation (Ornstein-Uhlenbeck process) using zero mean-flow and diffusion constants, applying only a single-point non-solenoidal forcing. The simulation results confirm the expected behavior.

Adding diffusion and mean-flow convection does not pose significant numerical solution problems. The solution corresponds very well with the expected analytical result, refer to Fig. 11(a).

Alternatively, also an explicit second order two-step stochastic Runge-Kutta method specifically suited for random source terms $(\text { SRK-2) })^{37}$ was applied. Simulation results with IE-2 compared very well with SRK-2 for simulations with flow and diffusion included. However, unexpected small time step limitations were observed during test simulations with SRK2 , significantly smaller than those expected from limitations posed by the viscous term, the reason yet unclear. Therefore, all later simulations were fully based on the implicit second order Euler method solved with simple over-relaxation and 10-20 sub-iterations (IE-2). Implicit time integration allowed an increase of the SRK-2 time step by a factor of up to $10^{3}$.

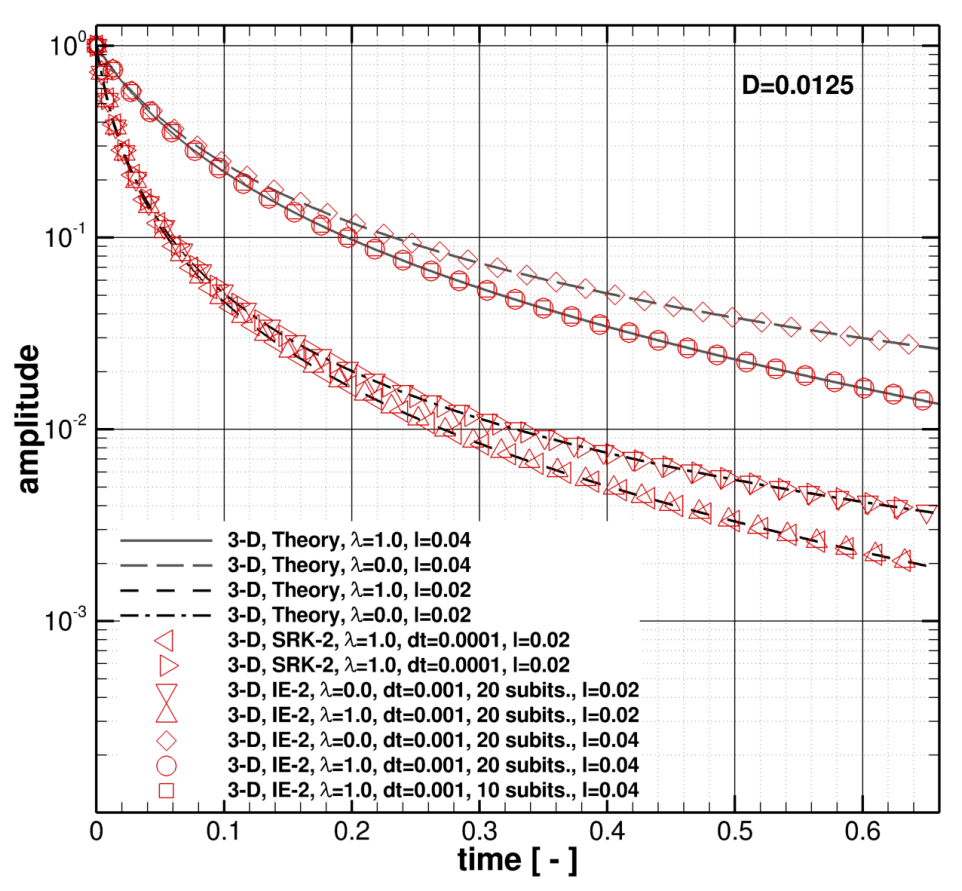

Figure 8. Decay test of initial disturbance with $(\lambda=1.0)$ and without $(\lambda=0.0)$ dissipation term in 2-D and 3-D and comparison with analytical solution; $D=0.0125, l_{0}=0.02$, mesh resolution $\Delta x=0.01$, time step $\Delta t=0.001$, implicit time integration with IE-2.

\section{Numerical Results}

\section{A. Validation of the Green function}

For a numerical test of the validity of the Green function derived in Section V.E.2 the initial value problem $v^{\prime}(\boldsymbol{x}, t=0)=\exp \left(-\left(\boldsymbol{x}-\boldsymbol{x}_{0}\right)^{2} / l_{0}^{2}\right)$ is simulated and compared to the analytical solution. The latter is 
found reformulating the homogeneous problem Eq. (61) with $Q=0$ into an inhomogeneous problem by introduction of a generalized variable for velocity $\widetilde{v}^{\prime}=H(t) v^{\prime}$,

$$
\frac{\partial \widetilde{v}^{\prime}}{\partial t}-D \frac{\partial^{2} \widetilde{v}^{\prime}}{\partial x_{j}^{2}}+\lambda \widetilde{v}=\delta(t) v^{\prime}(\boldsymbol{x}, t=0) .
$$

Eventually, convolution of the right-hand side source term with the Green function provides the analytical solution. Fig. 8 compares the decay behavior measured in the origin at $\boldsymbol{x}=0$ for the initial value problem $\left(D=0.0125, l_{0}=0.02\right.$, mesh resolution $\Delta x=0.01$, time step $\Delta t=0.001$, time integration with IE-2) from numerical solution and from convolution with the analytical Green function for a 2-D and a 3-D problem. The dissipation constant is varied between $\lambda=0$ (no dissipation, pure diffusion) and $\lambda=1.0$. Adding dissipation creates an increased decay of the signal. Excellent agreement between the analytical and the numerical solution is obtained.

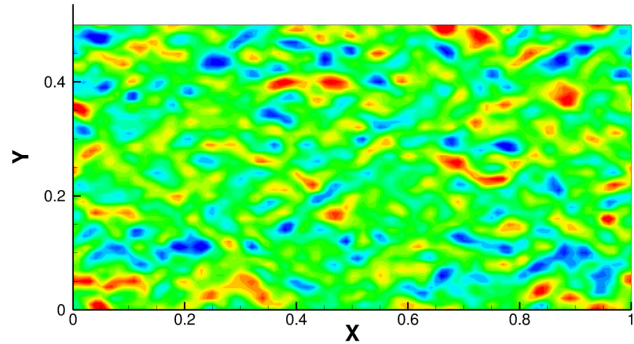

(a) Velocity component $v_{1}^{\prime}$, solenoidal forcing (SF).

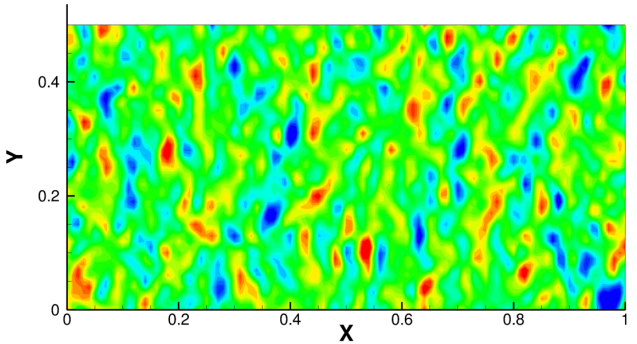

(b) Velocity component $v_{2}^{\prime}$, solenoidal forcing (SF).

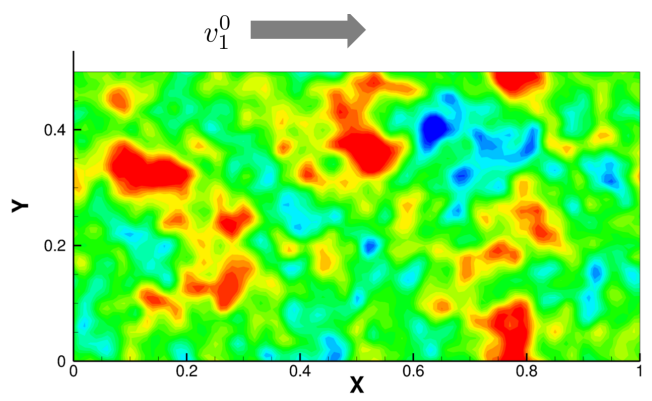

(c) Velocity component $v_{1}^{\prime}$, non-solenoidal forcing (NSF).

Figure 9. Snapshots of 2-D simulations, $D=0.02, \lambda=0.5, v_{1}^{0}=1.0, \alpha=v_{1}^{0} /\left(l_{s} \lambda\right)=10.0, l_{s}=0.2, l=0.01, \Delta x=0.01$.

\section{B. Homogeneous isotropic turbulence, constant mean flow}

First numerical simulations are carried for the forced advection-diffusion-dissipation equation Eq. (1) in 3-D and 2-D. Two types of vector forces are considered, viz (i) non-solenoidal forcing (NSF) based on mutually uncorrelated Gaussian correlated forcing terms as well as (ii) solenoidal (divergence-free) stochastic forcing (SF) defined by the curl of a stochastic forcing vector. The linear advection-diffusion-dissipation equation is solved implicitly in time as discussed in Section VI. The forcing is realized with the FRPM method based on a single Gaussian filter kernel of fixed spatial length scale $l$ resolvable on the applied mesh.

Fig. 9 shows snapshots of turbulent velocity fluctuations realized in 2-D on a $101 \times 51$ mesh. The nondimensional simulation parameters are indicated in the figure caption, where $D$ is the diffusion constant, $\lambda$ the inverse time scale. Uniform convection at velocity $v_{1}^{0}$ are realized. The turbulence decay ('slowness') parameter $\alpha=v_{1}^{0} /\left(l_{s} \lambda\right)=117.85$ indicates the ratio of turbulent decay time scale realized (defined by the parameter $1 / \lambda$ and convection time-scale $l_{s} / v_{1}^{0}$ ). The Cartesian mesh has uniform spacing $\Delta x$. Figures $9(\mathrm{a})$ and (b) show results obtained for solenoidal forcing, Figure 9(c) presents one velocity component with non-solenoidal forcing. Fig. 10 shows a snapshot from a 3-D simulation on a $101 \times 51 \times 51$ mesh using non-solenoidal forcing. 


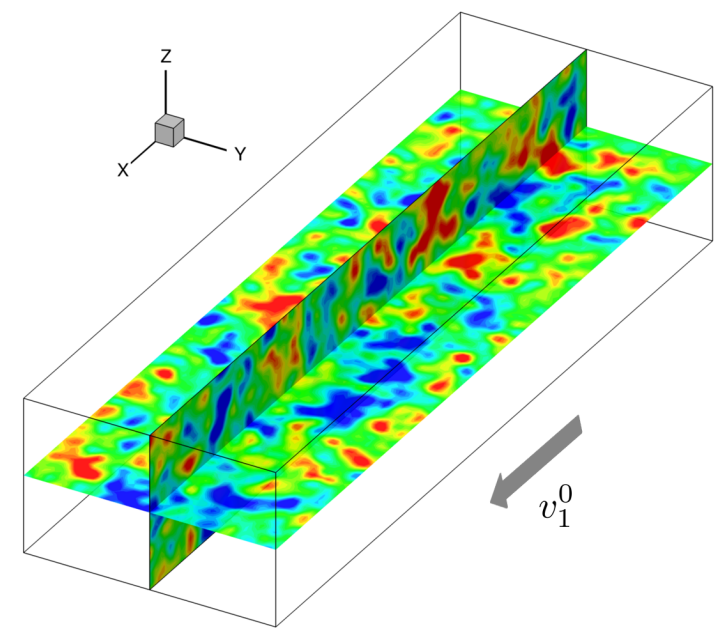

(a) Velocity component $v_{1}^{\prime}$.

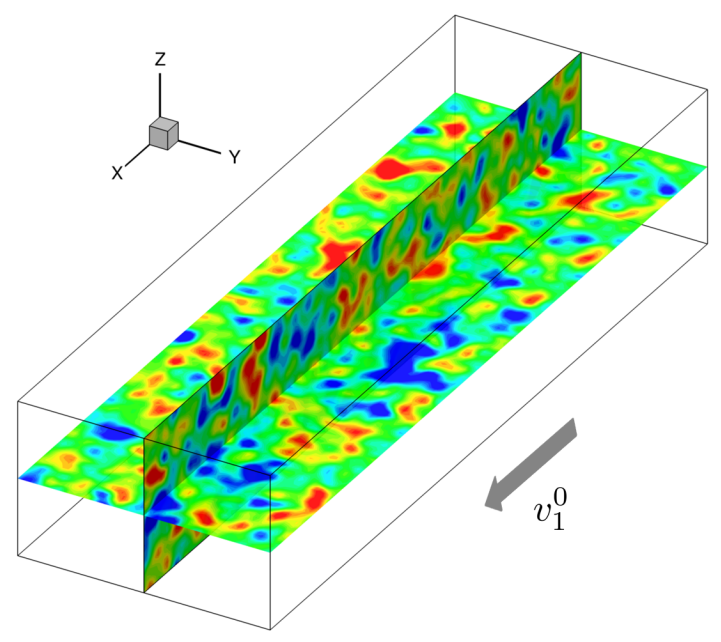

(b) Velocity component $v_{2}^{\prime}$.

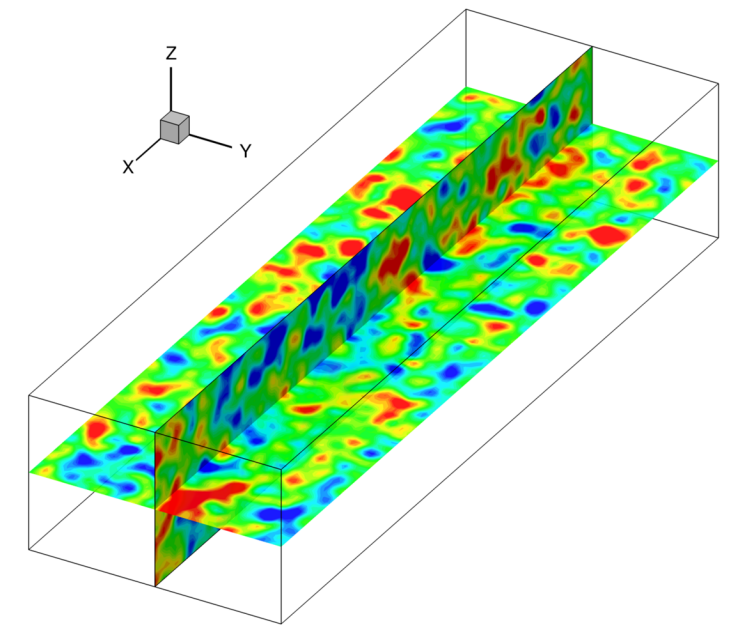

(c) Velocity component $v_{3}^{\prime}$.

Figure 10. Snapshot of fluctuating velocity, 3-D simulation, non-solenoidal forcing, $D=3 \cdot 10^{-4}, \lambda=0.06$, $v_{1}^{0}=0.50, \alpha=v_{1}^{0} /\left(l_{s} \lambda\right)=117.85, l_{s}=0.0707, l=0.02, \Delta x=0.01$.

Table 1. Numerical decay exponent $n$ of different 2-D and 3-D realizations

\begin{tabular}{ccc|cccc|ccc|c}
\hline \hline case & dimension & forcing & $D$ & $\lambda$ & $v_{1}^{0}$ & $l$ & $l_{s}=\sqrt{D / \lambda}$ & $\alpha=v_{1}^{0} /\left(l_{s} \lambda\right)$ & $n$ \\
\hline 2nd_st06 & 3-D & NSF & 0.005 & 0.02 & 1.00 & 0.01 & 0.5000 & 100.0 & -1.67 \\
2nd_st07 & 2-D & SF & 0.005 & 0.02 & 1.00 & 0.01 & 0.5000 & 100.0 & -1.25 \\
2nd_st08 & 2-D & SF & 0.005 & 0.02 & 1.00 & 0.01 & 0.5000 & 100.0 & -1.25 \\
st28 & 2-D & NSF & 0.00625 & 2.50 & 1.00 & 0.01 & 0.0500 & 8.0 & -1.85 \\
\hline \hline
\end{tabular}

The numerical results reveal that an explicit dissipation term in the forced linear equations is needed along with a diffusive term to obtain a characteristic low-pass shape of the turbulence power spectrum, i.e. a plateau for lower frequencies, and a characteristic roll-off that obeys a frequency power law. The crossover frequency is defined by the time scale introduced by the explicit dissipation term. Furthermore, for 


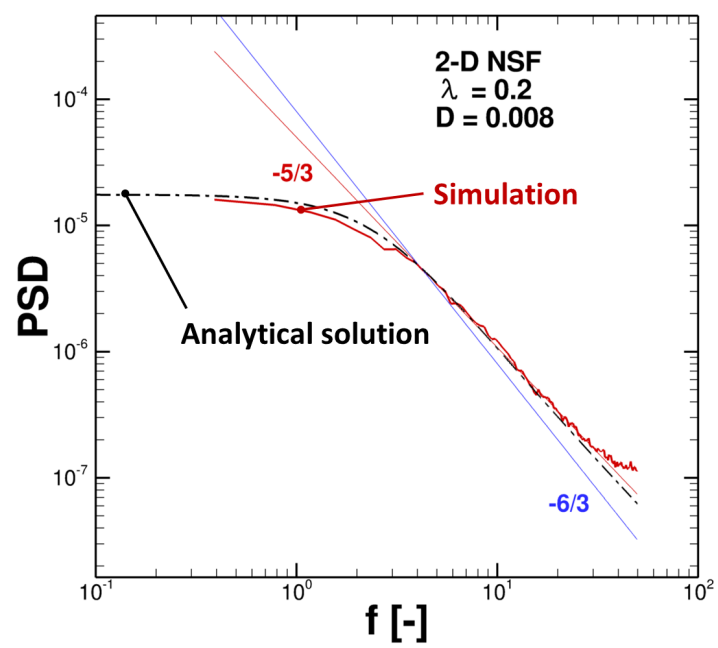

(a) Analytical versus computed frequency spectrum.

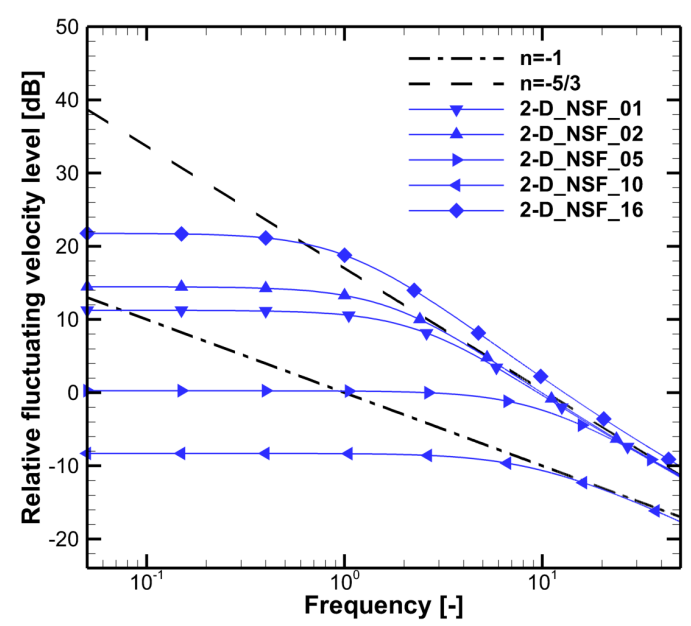

(b) Analytical velocity spectrum of cases Tab. 2.

Figure 11. 2-D analytical and numerical results for non-solenoidal forcing.

Table 2. Analytical cases discussed

\begin{tabular}{c|ccc|cc}
\hline \hline case & $D[-]$ & $\lambda[-]$ & $v_{1}^{0}[-]$ & $\alpha=v_{1}^{0} /\left(l_{s} \lambda\right)[-]$ & $l_{s}[-]$ \\
\hline 2-D_NSF_01 & 0.008 & 0.20 & 1.0 & 25.00 & 0.2 \\
2-D_NSF_02 & 0.008 & 0.10 & 1.0 & 35.36 & 0.2828 \\
2-D_NSF_05 & 0.005 & 2.00 & 1.0 & 10.00 & 0.05 \\
2-D_NSF_10 & 0.050 & 5.00 & 1.0 & 2.00 & 0.2 \\
2-D_NSF_16 & 0.005 & 0.02 & 1.0 & 100.00 & 0.5 \\
\hline \hline
\end{tabular}

homogeneous turbulence in equilibrium, the integral length scale is obtained as the square-root of diffusion constant times the dissipation time scale. The turbulent power law depends on the type of forcing term applied, the dimension of the problem, and the explicit value of the cut-off length scale $l$ chosen. For nonsolenoidal 2-D forcing a power law with exponent $n=-1.85$ to -2.0 is found. For 3-D non-solenoidal forcing the power law yields values of the exponent around $n=-5 / 3$. A solenoidal forcing in general provides a less pronounced decay with exponent around $n=-1.25$ in 2-D, refer to Tab. 1 for an overview about characteristic simulation results. Note, the first block of numbers indicates parameters set at the beginning of the simulation. The second block indicates parameters derived from the input values, i.e. the turbulence decay parameter $\alpha$ and the resulting integral length scale $l_{s}$. The last column presents spectral decay exponents found from the simulations.

The results are in general good agreement with the expected theoretical characteristics.

\section{Summary and Conclusions}

An advection-diffusion-dissipation equation with stochastic forcing was studied in this paper as an alternative way to set up stochastic sound sources for applications in computational aeroacoustics. The output are fluctuating velocity components, from which vortex sound sources derive. The model provides spectra and anisotropic two-point correlations that otherwise have to be incorporated in datum stochastic methods as additional model assumptions.

The stochastic partial differential equation, Eq. (1), involves a diffusion parameter, a time-scale, a redistribution tensor, and a forcing variance. It was shown that based on the defined form, the corresponding 
transport equations for turbulent kinetic energy and the Reynolds stresses comply with the canonical form of major RANS transport equations. The proper definition of the parameters from RANS transport equations was derived enabling an accurate reproduction of the RANS one-point statistics.

Two types of forcing are discussed, viz i) solenoidal forcing based on the curl of the stochastic source term and ii) non-solenoidal forcing based on a direct application of the stochastic forcing. For homogeneous isotropic flow, solenoidal forcing provides also solenoidal velocity fluctuations and reproduces in shape the cross-correlation tensor of isotropic homogeneous turbulence. The proper variance of the forcing term that is needed to reproduce a specific turbulent production rate is derived.

Theoretical frequency and wave-number spectra are discussed for homogenous turbulence. It is found that in 3-D a simple point forcing does not provide a useful exponent of the resulting wave-number spectrum. However, from the theoretical findings two alternative ways are identified to calibrate one-dimensional wavenumber and frequency spectra having the desired power law roll-off. First, consecutive application of two sets of F.L.A.D.D. equations with solenoidal point forcing yields an exponent around '-2'. Alternatively, a source term with calibrated wave-number spectrum can be used. The stochastic realization of the forcing wave number spectrum -Eq. (91)- is discussed in another paper on the conference. ${ }^{38}$ The generated integral length scale is defined by the dissipation time and the diffusion constant. Its magnitude matches very well with the magnitude from literature.

Numerical simulation results obtained with the procedure discusses are presented. Good agreement between numerical derived frequency spectra and the theoretical expected results demonstrate the benign behavior of the stochastic differential equation system. In particular, the results from consecutive F.L.A.D.D. application confirm the realization of proper spectra with point forcing. For the generation of two-point statistics, the hypothesis from turbulence modeling is adopted that the present model calibrated for homogeneous isotropic turbulence is also applicable for more general flows. Simulations for inhomogeneous flow will be conducted in ongoing work.

\section{Appendix}

\section{A. Additional FRPM features}

1. Cross-correlation function

For homogeneous synthetic turbulence the cross-correlation function

$$
R_{i j}(\boldsymbol{r}, \tau)=\left\langle q_{i}(\boldsymbol{x}, t) q_{j}(\boldsymbol{x}+\boldsymbol{r}, t+\tau)\right\rangle
$$

that results from the FRPM procedure outlined in the previous paragraphs derives ${ }^{8}$

$$
R_{i j}(\boldsymbol{r})=c \exp \left(-|\tau| / \tau_{s}\right) R(\boldsymbol{r}) \delta_{i j},
$$

where $R$ is the self-convolution $G \star G$ of the Gaussian Eq. (12), that reads

$$
R(\boldsymbol{r})=l^{d} \exp \left(-\frac{\pi|\boldsymbol{r}|^{2}}{4 l^{2}}\right)=C(r)
$$

Here $d$ denotes the dimension of the problem.

\section{Solenoidal velocity and HIT}

Solenoidal (divergence-free) fluctuating velocity components with $\partial v_{i} / \partial x_{i}=0$ can be generated from the curl of the vector generated by means of Eq. (10) (i.e. utilizing it as a vector potential),

$$
v_{i}^{\prime}=\epsilon_{i j k} \frac{\partial q_{k}}{\partial x_{j}} .
$$

It was discussed in Ref. ${ }^{7}$ that the such defined velocity fluctuations realize the two-point velocity correlation tensor $\mathcal{R}_{i j}=\left\langle v_{i}^{\prime}(\boldsymbol{x}, t) v_{j}^{\prime}(\boldsymbol{x}+\boldsymbol{r}, t+\tau)\right\rangle$ of homogeneous isotropic turbulence (HIT),

$$
\mathcal{R}_{i j}(\boldsymbol{r}, \tau)=v_{t}^{2}\left[\frac{f(r)-g(r)}{r^{2}} r_{i} r_{j}+g(r) \hat{\delta}_{i j}\right],
$$


where $r=|\boldsymbol{r}|$ and $r_{i}$ indicates a component of $\boldsymbol{r}$ and $v_{t}^{2}=\left\langle v_{i}^{\prime} v_{i}^{\prime}\right\rangle / 3$ is the turbulence intensity. It was shown in Ref. ${ }^{7}$ that in 3-D the lateral correlation function resulting from FRPM is related to the longitudinal correlation function through

$$
g(r)=f(r)+\frac{r}{2} \frac{\mathrm{d} f(r)}{\mathrm{d} r} .
$$

For the definition of longitudinal and lateral correlation functions refer e.g. to Pope ${ }^{23}$ or Batchelor. ${ }^{32}$ With a Gaussian filter function Eq. (12) a Gaussian longitudinal correlation function follows,

$$
f(r)=\exp \left(-\frac{\pi}{4} \frac{r^{2}}{l^{2}}\right)
$$

The integral length scale of it is $l$. For the realization of the proper turbulence intensity, the variance $c$ in Eq. (15) was derived as ${ }^{8}$

$$
c=\frac{2^{3-d} l^{2} v_{t}^{2}}{\pi}
$$

The wave-number spectrum generated by FRPM is a Gaussian of the form

$$
E_{G}(k, l)=\frac{4 v_{t}^{2}}{\pi^{3}} k^{4} l^{5} \exp \left(-\frac{k^{2} l^{2}}{\pi}\right) .
$$

\section{Generalized turbulence spectra}

In recent work the generation of generalized turbulence spectra has been discussed by Rautmann et al. ${ }^{25}$ and Wohlbrandt et al. ${ }^{24}$ Analytical expressions have been derived from the Gaussian transformation proposed by Alecu $^{39}$ to prescribe standard turbulence spectra through the superposition of Gaussian spectra. The transforms have been given for a Liepmann spectrum, ${ }^{25}$ as well as the von Kármán and modified von Kármán spectrum. ${ }^{24}$ The target spectrum is derived from the integral transformation over Gaussian turbulence spectra with variable length scale,

$$
E(k, \Lambda)=\int_{0}^{\infty} h(l, \Lambda) v_{t}^{2} e_{G}(k, l) \mathrm{d} l .
$$

Here, $e_{G}(k, l)=E_{G}(k, l) / v_{t}^{2}$ denotes a normalized Gaussian spectrum of length scale $l$, given by

$$
e_{G}(k, l)=\frac{4}{\pi^{3}} k^{4} l^{5} \exp \left(-\frac{k^{2} l^{2}}{\pi}\right) .
$$

Furthermore, $h(l, \Lambda)$ is the generating function (Gaussian transform) that defines the contribution of a Gaussian spectrum of length scale $l$. In addition, $\Lambda$ denotes the integral length scale of the target spectrum that could be spatially variable. For example, a Liepmann spectrum

$$
E_{L}(k, \Lambda)=\frac{8 v_{t}^{2}}{\pi} \frac{k^{4} \Lambda^{5}}{\left(1+k^{2} \Lambda^{2}\right)^{3}}
$$

is obtained from the generating function ${ }^{25}$

$$
h(l, \Lambda)=\frac{2}{\pi \Lambda} \exp \left(-\frac{l^{2}}{\pi \Lambda^{2}}\right) .
$$

For a numerical realization with FRPM, the integral Eq. (109) is discretized by a summation over discrete length scales $l_{m}$ with increment $\Delta l_{m}$,

$$
E(k, \Lambda)=\sum_{m} h\left(l_{m}, \Lambda\right) \Delta l_{m} v_{t}^{2} e_{G}\left(k, l_{m}\right) .
$$

Each length scale $l_{m}$ is provided by one FRPM realization. Based on the scaling Eq. (107) applied with $l=l_{m}$ and $v_{t}^{2}$ each FRPM realization provides fluctuations with turbulence intensity $v_{t}^{2}$. From Eq. (113) 
it infers that each FRPM realization furthermore must be scaled with an additional factor $\sqrt{h\left(l_{m}, \Lambda\right) \Delta l_{m}}$ such that the energies are summed up correctly.

In order to achieve a good numerical approximation, Wohlbrandt et al. ${ }^{24}$ propose to use an exponential distribution of the length scales

$$
l_{m}=l_{0} q^{m}, \quad q=\left(\frac{l_{M}}{l_{0}}\right)^{\frac{1}{M}}, \quad m \in\{0 \ldots M\},
$$

where $l_{0}$ and $l_{M}$ indicate the smallest and largest resolved length scales, respectively. Using the trapezoidal rule for numerical integration, the length scale increments become $(p \in\{2 \ldots M-1\})$

$$
\Delta l_{0}=\frac{l_{1}-l_{0}}{2}, \quad \Delta l_{p}=\frac{l_{p+1}-l_{p-1}}{2}, \quad \Delta l_{M}=\frac{l_{M}-l_{M-1}}{2} .
$$

This implies the following numerical procedure to proceed from time $t^{n}$ to $t^{n+1}$ : Let

$$
\varphi_{m}:=\sqrt{h\left(l_{m}, \Lambda\right) \Delta l_{m}}, \quad \text { and } \quad G_{m}:=G\left(r, l_{m}\right),
$$

with $G$ defined by Eq. (12). Then,

- move particles from position at time level $n$ to new one at time level $n+1$,

- generate with Eq. (19) $M+1$ mutually uncorrelated random variables $r_{i k}^{n+1, m}$ (indicated by an additional upper case index $m, m \in\{0 \ldots M\})$,

- compute the solution at new time level via weighted superposition of discrete convolutions defined in Eq. (17),

$$
q_{i k}^{n+1}=\sum_{m} \varphi_{m} G_{m} \widehat{\star} r_{i k}^{n+1, m},
$$

- compute velocity fluctuations from Eq. (103).

Note, so that energies sum up correctly in correspondence with Eq. (113) the $M+1$ different FRPM realizations must be mutually uncorrelated, i.e.,

$$
\left\langle r_{i k}^{n, m} r_{i k}^{n, l}\right\rangle=\delta_{m l}
$$

This implies to use source terms in Eq. (19) that are uncorrelated for different length-scale realizations, i.e.,

$$
\left\langle\sigma_{i k}^{n, m} \sigma_{i k}^{n, l}\right\rangle=\delta_{m l}
$$

\section{Acknowledgement}

The author expresses his thanks to Christina Appel and Attila Wohlbrandt for providing RANS simulations for the turbulent boundary layer and NACA 0012 inflow test case (to be used). Financial support is provided by the German Research Foundation (Deutsche Forschungsgemeinschaft, DFG) in the framework of the Sonderforschungsbereich 880 .

\section{References}

\footnotetext{
${ }^{1}$ Kraichnan, R., "Diffusion by a random velocity field," Physics of Fluids, Vol. 13, 1970, pp. 22-31.

${ }^{2}$ Bechara, W., Bailly, C., Lafon, P., and Candel, S., "Stochastic approach to noise modelling for free turbulent flows," AIAA Journal, Vol. 32, No. 3, 1994, pp. 455-463.

${ }^{3}$ Bailly, C., Lafon, P., and Candel, S., "Computation of noise generation and propagation for free and confined turbulent flows," AIAA 1996-1732, State College, Pennsylvania, 1996.

${ }^{4}$ Bailly, C. and Juve, D., "A stochastic approach to compute subsonic noise using linearized Euler's equations," $A I A A$ 1999-1872, Greater Seattle, Washington, 1999.

${ }^{5}$ Ewert, R. and Emunds, R., "CAA Slat Noise Studies Applying Stochastic Sound Sources Based On Solenoidal Digital Filters," AIAA Paper 2005-2862, 2005.
} 
${ }^{6}$ Ewert, R., "RPM - the fast Random Particle-Mesh method to realize unsteady turbulent sound sources and velocity fields for CAA applications," AIAA Pap. 2007-3506, 2007.

${ }^{7}$ Ewert, R., "Broadband slat noise prediction based on CAA and stochastic sound sources from a fast random particle-mesh (RPM) method," Computers \& Fluids, Vol. 37, 2008, pp. 369-387.

${ }^{8}$ Ewert, R., Dierke, J., Siebert, J., Neifeld, A., Appel, C., Siefert, M., and Kornow, O., "CAA broadband noise prediction for aeroacoustic design," Journal of Sound and Vibration, Vol. 330, No. 17, 2011.

${ }^{9}$ Kozubskaya, T., Abalakin, I., and Bobkov, V., "A half-stochastic model for noise in free turbulent flows," 7th AIAA/CEAS Aeroacoustics Conference and Exhibit. Maastricht,Netherlands, 2001, AIAA Pap. 2001-2258.

${ }^{10}$ Billson, M., Eriksson, L.-E., and Davidson, L., "Jet noise prediction using stochastic turbulence modeling," AIAA 20033282, Hilton Head, South Carolina, 2003.

${ }^{11}$ Kamruzzaman, M., Lutz, T., Herrig, A., and Kraemer, E., "Semi-Empirical Modeling of Turbulent Anisotropy for Airfoil Self Noise Prediction," AIAA Journal, Vol. 50, No. 1, 2012, pp. 46-60.

${ }^{12}$ Kamruzzaman, M., Study of Turbulence Anisotropy and its Impact on Flow Induced Noise Emission, Doctoral thesis, Institute of Aerodynamics and Gas Dynamics, Faculty of Aerospace Engineering and Geodesy, University of Stuttgart, Stuttgart, Germany, 2011.

${ }^{13}$ Karabasov, S., Afsar, M., Hynes, T., Dowling, A., McMullan, W., Pokora, C., Page, G., and McGuirk, J., "Jet Noise: Acoustic Analogy Informed by Large Eddy Simulation," AIAA Journal, Vol. 48, No. 7, 2010, pp. 1312-1325.

${ }^{14}$ Blake, W. K., "Mechanics of Flow-Induced Sound and Vibration, Complex Flow-Structure Interactions," Applied Mathematics and Mechanics, Vol. 17, No. II, 1986, pp. 756-767, Academic Press, Inc., Orlando, FL.

${ }^{15}$ Parchen, R., "Progress Report DRAW: A Prediction Scheme for Trailing Edge Noise Based on Detailed Boundary-Layer Characteristics," Report of the TNO (The Netherlands Organization) Institute of Applied Physics HAG-RPT-980023, Feb. 1998.

${ }^{16}$ Wilcox, D. C., Turbulence Modeling for CFD, DCW Industries, Inc., La Cañada, California, 3rd ed., 2006.

${ }^{17}$ Rotta, J., Turbulente Strömungen, B.G. Teubner Stuttgart, 1972.

${ }^{18}$ Wohlbrandt, A., "Das Random-Particle-Mesh Verfahren angewendet auf Schaufelvorderkantenlrm," Fachausschusssitzung zur Strömungsakustik von DGLR, DEGA und europischem X-Noise EV-Netzwerk, Munich, Nov. 2013.

${ }^{19}$ Siefert, M. and Ewert, R., "Sweeping Sound Generation in Jets Realized with a Random Particle-Mesh Method," AIAA Pap. 2009-3369, 2009.

${ }^{20}$ Ewert, R., Dierke, J., Appel, C., and Herr, M., "RANS/CAA based prediction of NACA 0012 broadband trailing edge noise and experimental validation," 15th AIAA/CEAS Aeroacoustics Conference, Miami, Florida, USA, 2009, AIAA Pap. 2009-3269.

${ }^{21}$ Ewert, R., Dierke, J., Appel, C., Pott-Pollenske, M., and Sutcliff, M., "CAA-RPM Prediction and Validation of Slat Setting Influence on Broadband High-Lift Noise Generation," 16th AIAA/CEAS Aeroacoustics Conference, Stockholm, Sweden, 2010, AIAA Pap. 2010-3833.

${ }^{22}$ Neifeld, A. and Ewert, R., "Jet Mixing Noise from Single Stream Jets using Stochastic Source Modeling," 17th AIAA/CEAS Aeroacoustics Conference, Portland, Oregon, USA, 2011.

${ }^{23}$ Pope, S., Turbulent Flows, Cambridge University Press, 2000.

${ }^{24}$ Wohlbrandt, A., Hu, N., Guerin, S., and Ewert, R., "Analytical reconstruction of isotropic turbulence spectra based on the Gaussian transform," Comp. E Fluids, Vol. 132, 2016, pp. 46-50.

${ }^{25}$ Rautmann, C., Dierke, J., Ewert, R., Hu, N., and Delfs, J., "Generic Airfoil Trailing-Edge Noise Prediction using Stochastic Sources from Synthetic Turbulence," AIAA Paper 2014-3298, 2014.

${ }^{26}$ Eisfeld, B., "Reynolds Stress Modeling for Complex Aerodynamic Flows," 5th European Conference on Computational Fluid Dynamics, ECCOMAS CFD, 14-17 Jun 2010, Lisbon, 2010.

${ }^{27}$ Al-Sharif, S. F., "Reynolds Stress Modeling for Complex Aerodynamic Flows," Computational Simulations and Applications, edited by J. Zhu, 2011, InTech, Available from: http://www.intechopen.com/books/computational-simulations-andapplications/reynolds-stress-transportmodelling.

${ }^{28}$ Rotta, J., "Statistische Theorie nichthomogener Turbulenz," Zeitschrift für Physik, Vol. 129, No. 6, 1951, pp. 547-572.

${ }^{29}$ Reiche, N., Lummer, M., Ewert, R., Delfs, J. W., and Moghadam, M. A., "Towards high-lift noise from Fast Multipole BEM with anisotropic synthetic turbulence sources." 21st AIAA/CEAS Aeroacoustics Conference, Dallas, United Stated of America., 2015, AIAA Pap. 2015-2672.

${ }^{30}$ Stefano, G. D. and Vasilyev, O. V., "Stochastic Coherent Adaptive LES of Forced Isotropic Turbulence," Direct and Large-Eddy Simulation VII, 2010, pp. 275-280, Volume 13 of the ERCOFTAC Series.

${ }^{31}$ Särkkä, S., "Applied Stochastic Differential Equations," Lecture Notes Version 1.0 (November 21, 2012), 2012.

${ }^{32}$ Batchelor, G., The Theory of Homogeneous Turbulence, Cambridge University Press, 1960.

${ }^{33}$ Ewert, R., Neifeld, A., and Wohlbrandt, A., "A three-parameter Langevin model for hot jet mixing noise prediction," AIAA Paper 2012-2238, 2012.

${ }^{34}$ Lesieur, M., Turbulence in Fluids, Springer, 2008.

${ }^{35}$ Hirt, C., Armsden, A., and Cook, J., "An arbitrary Lagrangian Eulerian computing method for all flow speed," Journal of Computational Physics, Vol. 135, 1997, pp. 203-216.

${ }^{36}$ Gardiner, C., Handbook of Stochastic Methods, Springer, 2004.

${ }^{37}$ Honeycutt, R. L., "Stochastic Runge-Kutta algorithmns. I. White noise," Physical Review A, Vol. 25, No. 2, 1992, pp. 600-603. 2016.

${ }^{38}$ Reiche, N. and Ewert, R., "Realization of Arbitrary Vorticity Spectra using Generic Stochastic Turbulence," Aiaa paper,

${ }^{39}$ Alecu, T. I., Voloshynovskiy, S., and Pun, T., "The Gaussian transform," EUSIPCO2005, 13th European Signal Processing Conference, 2005. 